Sharif University of Technology
Scientia Iranica
Transactions B: Mechanical Engineering
IRAN

\title{
Cooperative search and localization of ground moving targets by a group of UAVs considering fuel constraint
}

\author{
H. Nobahari*, M. Effati, and M. Motie \\ Department of Aerospace Engineering, Sharif University of Technology, Zip Code 1458889694, Tehran, Iran.
}

Received 13 March 2017; received in revised form 26 February 2018; accepted 17 December 2018

KEYWORDS
UAV;
Cooperative search
and localization;
Moving ground target;
Fuel constraint;
Extended Kalman
filter.

\section{Introduction}

Cooperative tasks of UAVs and robots without human control and guidance have always been challenging and exciting. The great number of research studies on cooperative algorithms, performed in recent years, shows the importance of this topic. Cooperative search and localization of the ground moving targets is one of the cooperative missions, mentioned for UAVs. Methods of target detection vary, as the sensors installed on UAVs are different. For instance, targets can be

\footnotetext{
*. Corresponding author.

E-mail addresses: nobahari@sharif.edu (H. Nobahari); meysameffati2014@gmail.com (M. Effati);

mahyar.motei@gmail.com (M. Motie)
}

doi: $10.24200 /$ sci. 2018.21186 monitored using vision, thermal or Radio Frequency (RF) sensors. This article focuses on search and localization of moving RF ground targets by a group of UAVs, when there is fuel constraint for UAVs, and the data, measured by the sensors, are noisy. This is one of the practical subjects in cooperative missions.

In recent years, cooperative search and localization of ground targets by a group of robots or UAVs has taken a great deal of attention. In [1], a group of robots observed an environment in the form of polygons. The problem is to ensure the visibility of the area by assigning an adequate number of robots and determining the proper position of sensors in the body of the robots. Cooperative observation of multiple moving targets by a group of robots was investigated in [2]; the focus was primarily on developing the distributed control strategies that allow the team to maximize the total time of observing the targets. In [3], 
path planning for cooperative localization of an $\mathrm{RF}$ target by a group of UAVs was investigated. Multitarget tracking by a group of heterogeneous vehicles (robots and UAVs) was presented in [4]. Ref. [5] is about surveillance of a field by a group of UAVs; in this paper, two objectives include minimizing the distance traveled by UAVs and maximizing the satisfaction of event viewers. Performance of GuidLoc, a localization system that includes a mini multi-rotor UAV and a directional antenna, in localization accuracy and distance traveled by the UAV was investigated in [6] In [7], localization of an RF emitter by a UAV was investigated; according to the received signal strength, the UAV decides how much is close to the target. In addition, in [8], the problem of localizing a ground moving target by a UAV using the EKF and UKF was investigated and compared together. Deghat et al. [9] investigated the localization of several targets by a single UAV; the localization method uses bearing-only measurement, and the proposed method, without using any filtering algorithm, allows tolerating measurement noises and slowing the movement of targets. In [10], a new algorithm (Range Parameterized Square Root Cubature Information Filter (RP-SRCIF)) for multiUAV passive localization of targets was investigated and compared to Unscented Kalman Filter (UKF) and Cubature Kalman Filter (CKF). The non-positive definiteness of error covariance in some cases results in the numerical instability of UKF and CKF, while the proposed algorithm does not have such a problem. In [11], a quadrotor was designed for target localization. Cooperative control of a team of robots for localization of a single target is the topic of [12]. Localization of a ground target by multiple heterogeneous unmanned vehicles (a team of aerial and ground vehicles) was the main problem in [13]. The problem in [14] involved localizing and tracking an RF target using EKF and a recursive Bayesian estimator. To predict the future path of the target, the steepest descent posterior CramerRao lower bound path planning and a bioinspired heuristic path planning were utilized, and the results were compared together. In addition, Shin et al. [15] investigated the persistent sensing of a ground moving target by a group of UAVs. The main contribution is the prediction of the future position of the target using the current information. In [16], localization of fixed-wing UAVs, when there is no GPS signal, using relative measurements of UAVs and known landmarks was investigated. In this work, Extended Kalman Filter (EKF) was used for a combination of the sensors' data and estimation of the required states of the UAVs.

Search, detection, and localization of targets can be done by a group of UAVs, equipped with different types of sensors such as downward looking millimeter wave radar [17], vision based [18-20], infrared (IR) [21], Radio Frequency (RF) [22-32], etc. In addition, a combination of two or more types of sensors can be utilized [33,34]. Since RF sensors are mentioned in this article, in the following, the research studies contain at least one RF sensor.

In [22], a set of UAVs searches for a stationary RF ground target in the presence of some obstacles. Optimal flight paths are planned so that UAVs can localize the target in a safe condition. In [23], cooperative search of a stationary RF ground target by multiple UAVs was investigated. UAVs search the target using the signal intensity, received from the target. A guidance algorithm is designed to optimize the search time.

In [24], a guidance law was developed to find a moving target by a group of UAVs. In addition, a cooperative Monte-Carlo estimator is merged with the guidance law to localize the target. In [25], search and localization of a lost target was investigated. Moreover, several neighborhood laws were developed, the effect of which on the search performance was investigated. The neighborhood laws introduce the UAVs that are about each UAV, and the UAVs cooperate with their neighbors.

In [26], the communication range and number of UAVs was modified to evaluate the performance of the cooperative search. In [27], a control architecture was presented that allows UAVs to cooperatively locate RF moving ground targets in the presence of noise. The control architecture includes four modes as Global Search (GS), Approach Target (AT), Locate Target (LT), and Target Reacquisition (TR). This control architecture was utilized in references [28-30], too. The goal of [28] was to find optimal paths in which a search cost function could be optimized. This function includes path length traveled by UAVs, search time, and fuel consumption. In [29], Kalman filter and triangulation techniques are utilized for cooperative localization of ground moving targets. These methods are compared in terms of time and accuracy of localization. Another method called angle-rate algorithm was proposed in [30].

Pack et al. [31] investigated the optimum position of sensors, type of sensors (all high-quality, all lowquality, or a random mixture of high- and low-quality sensors), and the optimum number of UAVs in order to achieve the highest accuracy of target localization. Although each UAV can localize the target separately, it was shown in [32] that the combination of several UAVs' measurements with different levels of accuracy improved the precision of localization. In [33], SigmaPoint Kalman Filter (SPKF) was utilized for cooperative localization of a moving target, when each UAV utilized one or more types of sensors between RF, IR, and image sensors.

There are several methods to decrease the time of search and enhance the accuracy of target localization. 
Hager et al. [34] utilized a combination of different vehicles and ground stations, equipped with different sensors, a ground robot equipped with IR sensors, two UAVs equipped with a camera, and four ground stations equipped with RF sensors. The simultaneous use of ground robots, UAVs, and ground stations is aimed at enhancing the accuracy and reducing the time of localization.

In this study, a group of UAVs with limited sensing capabilities is utilized to search and localize moving ground RF emitters in a cooperative fashion. During the search, UAVs perform the fueling process using a flying fuel tanker, whenever they need fuel. Moreover, it is assumed that each emitter moves with a constant speed and sends intermittently emitting signals. UAVs use the bearing-only measurements of the targets. The proposed search and localization algorithm have four known modes GS, AT, LT, and TR, as proposed in [28-30], as well as two new operating modes, called Approach Fuel Tanker (AFT) and fueling (FUE). In the GS mode, the search area is divided into several zones, and a selection function is proposed for selecting the appropriate zone as the next destination. This function depends on the number of times a zone is passed through, the time passed from the last visit of the zone, the average distance of the zone from UAVs, and the maneuver required for the UAV to turn toward the zone. In the LT mode, a new guidance law is proposed to perform a circular motion around the target. In this mode, the target is localized using the Extended Kalman Filter (EKF) rather than linear Kalman Filter (KF), which was used in [25-27]. In the TR mode, a new idea is proposed to increase the radius of circular motion. This method allows a UAV to increase the turning radius with a minimum maneuver effort. Furthermore, since UAVs have limited fuel, the search and localization algorithm is developed regarding this constraint. Accordingly, UAVs take turns for fueling using a turn-taking function. This function depends on the amount of the remaining fuel, the distance of UAV from the fuel tanker, and the number of UAVs in the queue. In addition, by using a function, UAVs decide to go to the AFT mode and, then, start their fueling. In this study, a ground control station is utilized for communication of UAVs with each other and the fuel tanker.

This paper is organized as follows. In Section 2, the problem is defined and the mathematical model of the vehicles (UAVs, moving ground targets, and fuel tanker) is presented. In Section 3, the architecture of the cooperative search and localization algorithm in the presence of fuel constraint is described. In this section, modes of flight and the conditions for switching between these modes are presented. In Section 4, the guidance algorithm utilized in each mode is described. In Section 5, the localization algorithm, by considering the information received from the UAVs sensors, is described. In Section 6, results of the simulated scenarios are presented. Finally, a conclusion is made in Section 7.

\section{Problem definition and mathematical models}

A group of UAVs, with limited sensing capabilities, cooperatively search and localize several ground mobile $\mathrm{RF}$ emitters in a rectangular area. Each emitter has a constant speed and sends intermittently emitting signals. UAVs are equipped with RF passive seekers. In other words, RF signals sent from the ground targets are detected by RF seekers, installed on the UAVs. UAVs search the area within the detectable range of their seeker. Since the extended Kalman filter is used for localization, UAVs perform a near circular motion around the targets to provide sufficient time for convergence of the estimated states to true ones. Another factor added to the problem is the fuel constraint; therefore, a flying fuel tanker is also considered.

\subsection{Equations of motion}

Assuming that the total angle-of-attack is small enough to neglect, the motion equations of each UAV in the local frame (Figure 1) are represented as follows [35]:

$$
\begin{aligned}
\ddot{x}_{U}= & a_{x, U} C \theta_{U} C \psi_{U}-a_{y, U} \\
& \left(S \phi_{U} S \theta_{U} C \psi_{U}+C \phi_{U} S \psi_{U}\right)-a_{z, U} \\
& \left(C \phi_{U} S \theta_{U} C \psi_{U}-S \phi_{U} S \psi_{U}\right)
\end{aligned}
$$

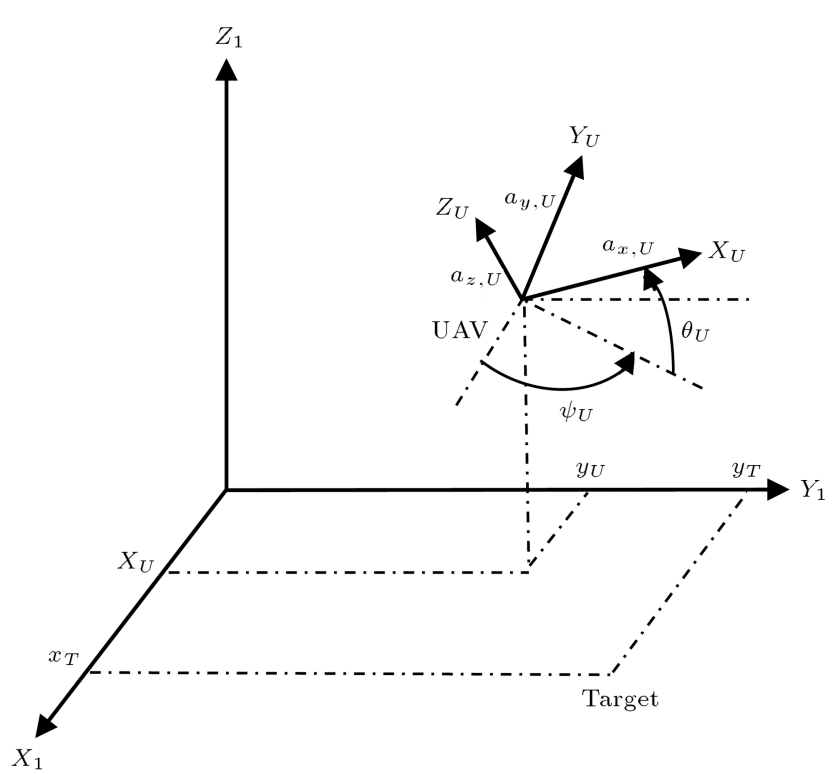

Figure 1. Three-dimensional UAV and target situation $\left(\left[X_{U}, Y_{U}, Z_{U}\right]\right.$ and $\left[X_{l}, Y_{1}, Z_{l}\right]$ are the axes of body and local coordinate systems, respectively). 


$$
\begin{aligned}
\ddot{y}_{U}= & a_{x, U} C \theta_{U} S \psi_{U}-a_{y, U} \\
& \left(S \phi_{U} S \theta_{U} S \psi_{U}+C \phi_{U} C \psi_{U}\right)-a_{z, U} \\
& \left(C \phi_{U} S \theta_{U} S \psi_{U}-S \phi_{U} C \psi_{U}\right), \\
\ddot{z}_{U}= & a_{x, U} S \theta_{U}+a_{y, U} S \phi_{U} C \phi_{U}+a_{z, U} C \phi_{U} C \theta_{U}-g, \\
\dot{\psi}_{U}= & \left(a_{y, U} C \phi_{U}-a_{z, U} S \phi_{U}\right) /\left(v_{U} C \theta_{U}\right), \\
\dot{\theta}_{U}= & \left(a_{y, U} S \phi_{U}+a_{z, U} C \phi_{U}-g C \theta_{U}\right) / v_{U},
\end{aligned}
$$

where $C$ and $S$ stand for math functions, cos and $\sin$, respectively. Moreover, $\left[x_{U}, y_{U}, z_{U}\right], g, \psi_{U}$, and $\theta_{U}$ are position of the UAV in the local frame, gravitational acceleration, yaw, and pitch angles, respectively. In addition, $a_{x, U}, a_{y, U}$, and $a_{z, U}$ are longitudinal and lateral accelerations, and $\phi_{U}$ is the roll angle in the body frame. To have a small side slip angle, UAVs are controlled such that $a_{y, U}=0$.

The motion equations of fuel tanker are the same as those represented in Eq. (1). In addition, the velocity of fuel tanker is constant. The ground targets start from $\left[x_{0, T}, y_{0, T}, 0\right]$ and move along the horizontal plane with the constant speed $\left[v_{x, T}, v_{y, T}, 0\right]$.

\subsection{Transfer functions}

Accelerations, generated by the guidance law of UAVs, are in body frame and denoted by $a_{x c, U}, a_{y c, U}$, and $a_{z c, U}$. The lateral commands, $a_{y c, U}$ and $a_{z c, U}$, are converted to $\phi_{c, U}$ and $a_{c z, U}$ as follows:

$$
\begin{aligned}
& a_{c z, U}=\operatorname{sign}\left(a_{z c, U}\right) \sqrt{a_{y c, U}^{2}+a_{z c, U}^{2}}, \\
& \phi_{c, U}=\operatorname{atan}\left(\frac{a_{y c, U}}{a_{z c, U}}\right) .
\end{aligned}
$$

However, according to Eq. (1), the point mass model uses $a_{x, U}, \phi_{U}$ and $a_{z, U}$. The transfer functions between the commanded and achieved accelerations and roll angle are typically assumed as follows:

$$
\begin{aligned}
\frac{a_{x, U}(s)}{a_{x c, U}(s)} & =\frac{1}{0.0100 s^{2}+0.140 s+1}, \\
\frac{a_{z, U}(s)}{a_{c z, U}(s)} & =\frac{1}{0.0091 s^{2}+0.133 s+1}, \\
\frac{\phi_{U}(s)}{\phi_{c, U}(s)} & =\frac{1}{0.0083 s^{2}+0.127 s+1} .
\end{aligned}
$$

Similarly, transfer functions of the fuel tanker are considered as follows:

$$
\begin{aligned}
\frac{a_{z, F T}(s)}{a_{c z, F T}(s)} & =\frac{1}{0.01 s^{2}+0.14 s+1}, \\
\frac{\phi_{F T}(s)}{\phi_{c, F T}(s)} & =\frac{1}{0.1 s+1} .
\end{aligned}
$$

\subsection{Altitude and velocity hold}

Since UAVs and fuel tanker should fly in certain altitudes, a simple altitude hold system is considered, the block diagram of which is depicted in Figure 2 .

In the figure, $h_{d}$ and $h$ are respectively the desired and achieved altitudes, $a_{z c, h}$ is the acceleration command in the vertical plane, and $k_{i h}(i=1,2)$ are gains. Since UAVs should change their speed in switching between different modes, a velocity control loop is required, the block diagram of which is depicted in Figure 3.

In the figure, $v_{d}$ and $v$ are respectively the desired and achieved velocities, $a_{x c}$ is the longitudinal acceleration command, and $k_{i v}(i=1,2)$ are gains.

\subsection{UAVs fuel model}

Each UAV has a fuel model based on its fuel consumption. UAV fuel model is as follows:

$$
f_{U}=f_{\max }-r_{F U} t
$$

where $r_{F U}$ and $f_{\max }$ are the fuel consumption rate $(\mathrm{kg} / \mathrm{sec})$ and the maximum fuel mass $(\mathrm{kg})$, respectively.

\subsection{Targets signal model}

It is assumed that each UAV has a seeker by which the direction and the strength of the RF signal, emitted by the target, are measured. The propagation ranges of the target signal are stated by $r_{S_{\max }}$ (detection range) and $r_{S_{c}}$ (tracking range). Herein, $r_{S_{\max }}$ is assumed as the maximum distance from the target where the seeker can detect the target and $r_{S_{c}}$ is the maximum distance from the target where the signal intensity is enough for the seeker to track and locate the target. The tracking range of the seeker is assumed to be less than the detection range. For example, when $r$ (distance of the UAV from the target) is less than $r_{S_{\max }}$, it is assumed that the UAV detects the target and goes to the AT mode. When $r$ becomes less than $r_{S_{c}}$, the UAV goes to the LT mode and starts to locate the target.

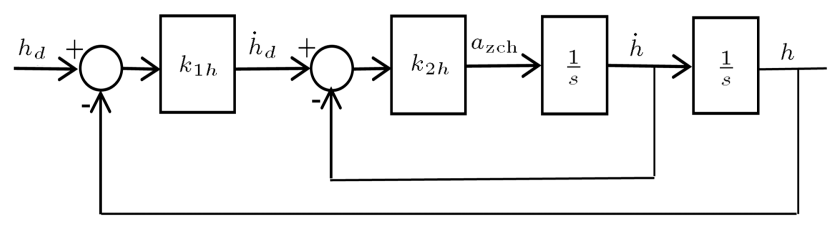

Figure 2. Block diagram of the altitude control loop.

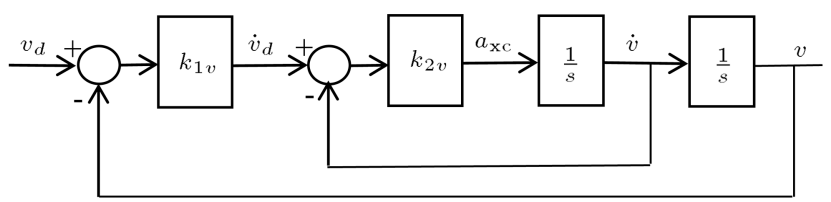

Figure 3. Block diagram of speed control loop. 


\section{Cooperative search and localization architecture}

Figure 4 shows the general architecture of the proposed cooperative search and localization system. UAVs and fuel tanker share their information through a global database (DB). A Ground Control Station (GCS) also communicates with database and performs some processes, the results of which are shared with UAVs through global database. UAVs use these data in their decisions, e.g., to select the next zone for search, etc. In the following, first, the data inserted to DB by UAVs, fuel tanker, and GCS, are introduced. Then, flight modes of UAVs and the switching criteria between different modes are explained.

\subsection{Database}

UAVs, fuel tanker, and GCS data are recorded in database. For this purpose, five tables entitled "UAVs history", "Fuel tanker", "Located targets", "Zones", and "Times" are utilized (Figure 5). These tables are described in the following. "UAVs history" table records UAV ID and UAV position components and the corresponding time. Each UAV sends this information to DB by a time period of $t_{u}$.

"Fuel tanker" table records the instantaneous position and velocity of FT and a binary variable,

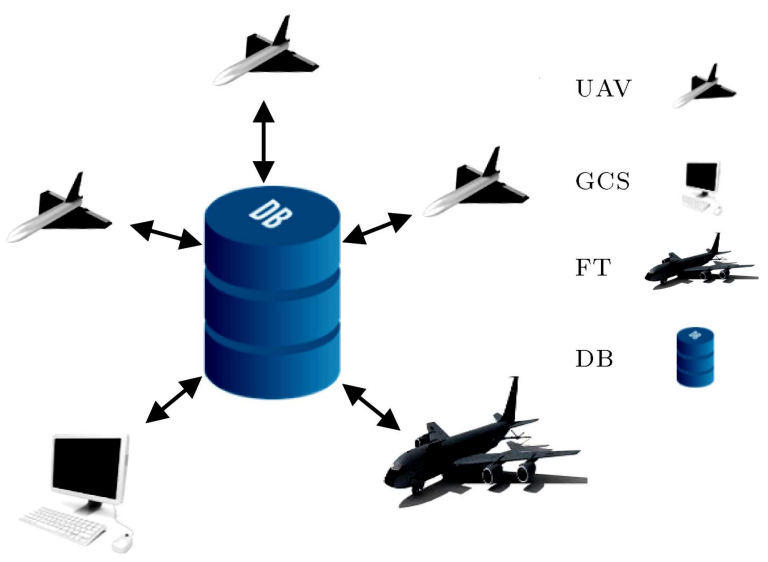

Figure 4. Indirect communication between UAVs, FT, and GCS through DB center.

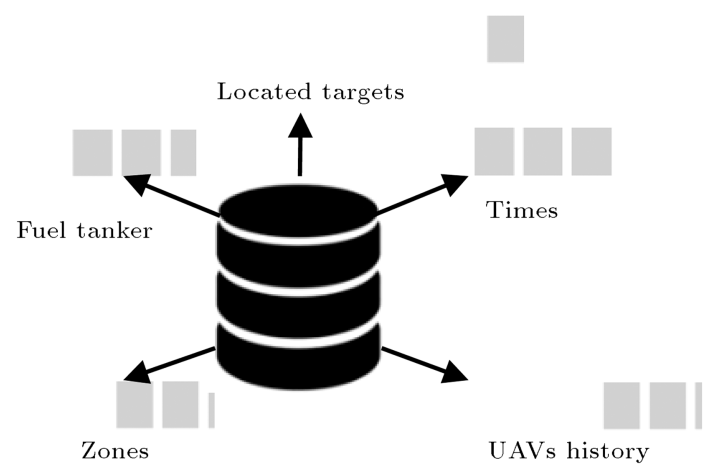

Figure 5. Tables of the database.

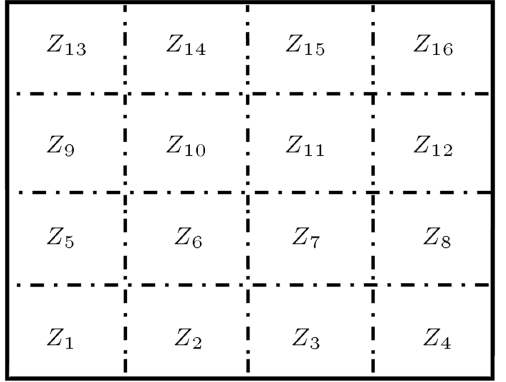

Figure 6. Division of the search area into zones.

called UF. This variable indicates whether any UAV is Under Fueling (UF) or not. UAVs use this variable to make a decision regarding fueling. Fuel tanker also sends its information to DB by the time period, $t_{u}$. "Located targets" table records $x$ and $y$ of the located targets and the localization time. These data are inserted to this table immediately after localization of a ground target.

As mentioned before, the search area is divided into some zones. For instance, 16 zones are shown in Figure 6. "Zones" table records the updated data of each zone, including the number of seen, time of last seen, and the average distance of the center of zone from UAVs.

"Times" Table records the turn-taking times for fueling, start time for moving toward the fuel tanker, and beginning and end time of fueling of each UAV.

\subsection{Guidance modes}

Figure 7 shows the decision diagram where the decision

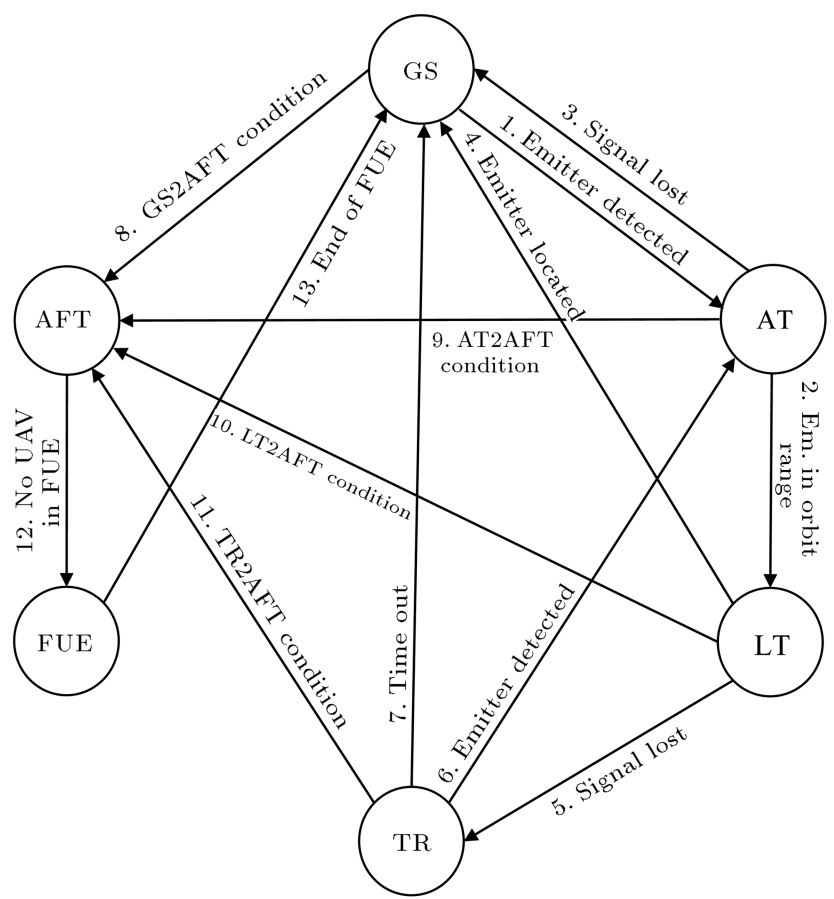

Figure 7. Decision diagram for the UAV mode selection in the presence of fuel constraint. 
to change from one mode to another is represented by arrows. The event, after which a mode is changed, is written behind the arrow. It should be noted that UAVs cooperate with each other only in GS, AFT, and FUE modes, and they operate independently in other modes.

In GS, each UAV evaluates a selection function for all zones in neighborhood. For example, if a UAV is in Zone 6 (Figure 6), the neighboring zones are as 1 , $2,3,5,7,9,10$, and 11 . The zone selection function is defined as follows:

$$
f_{s}=w_{1} n_{s}+w_{2} \frac{1}{t_{s}}+w_{3} \frac{1}{\bar{d}}+w_{4}\left|\Delta \varphi_{U}\right|,
$$

where $w_{i}(i=1, \ldots, 4)$ are constant weights, $n_{s}$ is the number of times a zone has passed through, $t_{s}$ is the time passed from the last visit of the zone, $\bar{d}$ is the average distance of the zone from UAVs, and $\Delta \varphi_{U}$ is the required change in heading to reach the center of the zone. It should be noted that the ground control station periodically updates $n_{s}, t_{s}$, and $\bar{d}$ using the "UAVs history" table and saves them in "zones" table. Then, each UAV calculates $\Delta \varphi_{U}$ by getting $n_{s}, t_{s}$, and $\bar{d}$ from the database, and calculates $f_{s}$ for each zone in the neighborhood. Then, the zone with minimum $f_{s}$ is selected as the next destination for search; afterwards, a point within the selected zone is chosen randomly, and UAV is guided toward it.

According to Figure 7, the operating mode of a UAV changes from GS to AT when it receives a signal from a target (Event 1 ). In this mode, the UAV approaches to the target in the Line Of Sight (LOS) direction. If the target signal is lost in the approach, the mode changes from AT to GS (Event 3).

According to Figure 7, when a UAV receives a signal with enough intensity, the operating mode changes from AT to LT (Event 2). In this mode, the UAV performs a circular motion around the target and localizes it. When the target is located, the operating mode changes from LT to GS (Event 4).

If the target signal is lost during the LT mode, the UAV mode changes from LT to TR (Event 5). In this mode, the UAV increases its radius of circular motion around the target. If the signal is again received from the target, the mode changes from TR to AT (Event 6). Otherwise, if the time, permitted for TR, is finished and there is no signal from the lost target, the operating mode changes from TR to GS (Event 7).

Since UAVs have a certain amount of fuel, they must perform fueling using an air fuel tanker. UAVs use the following decision function when they are going to take turn for fueling:

$$
f_{T T}=w_{1} p_{F}-w_{2}\left(\frac{n}{n_{U}-1}\right)-w_{3}\left(\frac{d_{F T}}{d_{F T, \max }}\right),
$$

where $p_{F}$ is the fuel percent (between 0 and 1), $n$ is the number of UAVs in turn, $n_{U}$ is the total number of
UAVs, $d_{F T}$ is the distance of UAV from the fuel tanker, $d_{F T, \text { max }}$ is the maximum probable distance from the fuel tanker, and $w_{i}(i=1,2,3)$ are the weights that control the trade-off between these terms. Since the normalized parameters and the weights in Eq. (7) are less than one, the maximum value of the turn-taking function is one.

Once $f_{T T}$ for a UAV becomes fewer than $f_{T T, C}$, the UAV takes turn for fueling. The lower bound $f_{T T, C}$ is calculated for the worse condition as follows:

$$
\begin{aligned}
& f_{T T, C}= \\
& w_{1} \frac{f_{\max }-\left(\left(n_{U}-1\right) t_{F U}+\frac{d_{F T, \max }}{v_{U}}\right) r_{F U}}{f_{\max }}-w_{2}-w_{3},
\end{aligned}
$$

where $r_{F U}$ and $f_{\max }$ are fuel consumption rate and maximum amount of fuel, respectively. According to Eq. (8), $f_{T T, C}$ is calculated in the worst condition when the UAV has the maximum distance from the fuel tanker, and all other UAVs are in fueling queue.

According to Figure 7, once the conditions for approaching the fuel tanker are satisfied, the UAV switches from GS, AT, LT or TR modes to AFT mode. These conditions are stated in the following: If there is no UAV in the queue, the operating mode changes to AFT. In addition, if a UAV is not in the first place in the fueling queue, it has still a chance to approach the fuel tanker under the condition $\Delta t_{g} \geq 0$. In other words, when there is a time gap $\left(\Delta t_{g}\right)$ between the arrival of the UAV to the fuel tanker and the fueling process of the previous UAV, which is in AFT mode, the UAV goes to the AFT mode. The arrival time of a UAV to the fuel tanker is approximated as follows:

$$
t_{A F T}=\frac{\bar{d}_{F T}}{v_{U}},
$$

where $\bar{d}_{F T}$ is the distance from the center of the fuel tanker loiter area (Figure 8), and $v_{U}$ is the velocity

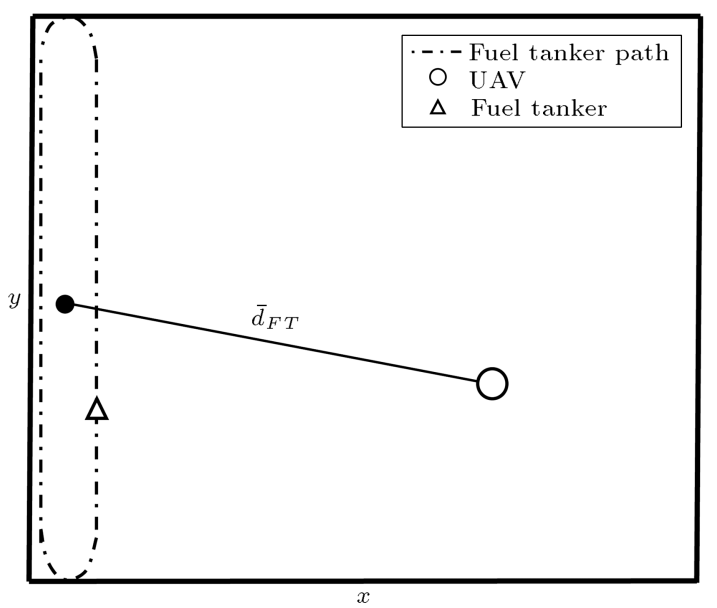

Figure 8. Fuel tanker loiter path and approximate distance of a UAV from the fuel tanker. 
of the UAV. Therefore, $\Delta t_{g}=t_{A F T}-\left(t_{A F T, P U}+t_{f u}\right)$ where $t_{A F T, P U}$ is the approximate time it takes to reach the fuel tanker for the previous UAV, which is in the AFT mode; $t_{f u}$ is the required fueling time of a UAV.

Thus, if one of the aforementioned conditions is established, a UAV switches from four modes of GS, AT, LT, and TR to the AFT mode (Events 8, 9, 10, and 11).

When a UAV is in the AFT mode, if it is the first UAV in the queue, it follows the fuel tanker in a certain distance until the fueling process of the previous UAV is finished. Then, the operating mode changes from AFT to FUE (Event 12), and the UAV becomes close to the fuel tanker and starts the fueling process. Once the fueling process is finished, the UAV operating mode changes from FUE to GS (Event 13).

\section{Formulation of the guidance algorithms}

Each UAV has six operating modes, namely GS, AT, LT, TR, AFT, and FUE. Each mode has its own guidance algorithm. In each mode, a virtual target is considered, the position of which is defined by mathematical relations. These virtual targets are used to guide the UAVs to perform the required maneuvers in each operating mode. It is important to note that all modes utilize a combination of True Proportional Navigation (TPN) and Velocity Pursuit (VP) guidance laws; they differ in the definition of the virtual target. In the VP guidance law, the objective is to keep the velocity vector toward the virtual target. The acceleration command, generated by these guidance laws, is calculated as follows:

$$
\begin{aligned}
& \mathbf{a}_{c-T P N}=N \boldsymbol{\omega} \times \mathbf{v}_{c}, \\
& \boldsymbol{\omega}=\frac{\mathbf{r}_{r} \times \mathbf{v}_{r}}{\left\|\mathbf{r}_{r}\right\|^{2}} \\
& \mathbf{a}_{c-V P}=\frac{k_{V P}\left(\mathbf{v}_{U} \times \mathbf{e}_{\mathrm{LOS}}\right) \times \mathbf{v}_{U}}{\left|\mathbf{v}_{U}\right|^{2}},
\end{aligned}
$$

where $N$ is the effective navigation ratio, $\omega$ is the angular velocity vector of the LOS, $\mathbf{v}_{U}$ is the velocity vector of the UAV, $\mathbf{v}_{c}$ is the closing velocity, $\mathbf{r}_{r}$ and $\mathbf{v}_{r}$ are the relative position and velocity vectors of the virtual target with respect to the UAV, $k_{V P}$ is the guidance coefficient of $\mathrm{VP}$, and $\mathbf{e}_{L O S}$ is the unit vector in the direction of target line-of-sight. In the following, the virtual target utilized in each operating mode is introduced.

\subsection{Global search}

In global search, the virtual target is a random position chosen within the selected zone. This position is sampled as follows:

$$
x_{V T}=x_{\mathrm{COZ}}+\frac{b}{2 n_{x}} r_{1}
$$

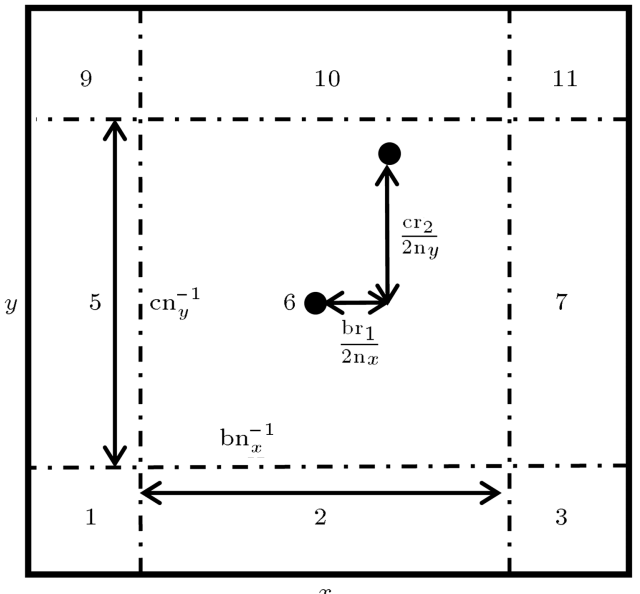

Figure 9. Selection of the virtual target in GS.

$$
y_{V T}=y_{\mathrm{COZ}}+\frac{c}{2 n_{y}} r_{2},
$$

where COZ stands for the center of the selected zone, $b$ and $c$ are respectively the length and width of the search area, $r_{1}$ and $r_{2}$ are random numbers in the range of $[-1,1]$, and finally $n_{x}$ and $n_{y}$ are number of the longitudinal and lateral divisions of the search area. For example, if Zone 6 is the selected zone (Figure 9), according to Eq. (12), each point of this zone has its chance to be chosen as the virtual target. Once UAV reaches near the randomly chosen point, it selects the next zone and the process is repeated.

\subsection{Approach target}

After detection of a ground target, the operating mode of the UAV changes from GS to AT. In the AT mode, the UAV moves along the LOS toward the target; in this mode, the UAV follows a virtual target along the LOS vector, slightly ahead of the UAV. The position of virtual target is calculated as follows:

$$
\mathbf{p}_{V T}=\mathbf{p}_{U}+l_{A T} \mathbf{e}_{\mathrm{LOS}},
$$

where $\mathbf{p}_{U}$ and $\mathbf{p}_{V T}$ are respectively the position vector of UAV and virtual target, and $l_{A T}$ is a constant that represents the distance between UAV and virtual target.

\subsection{Locate target}

When target signal becomes strong enough, the operating mode changes from AT to LT. The target position is unknown, and the passive seeker measures only the target LOS angles. The idea, proposed to perform a circular motion, is to move along a horizontal vector perpendicular to the LOS. Therefore, the position of the virtual target is calculated as follows:

$$
\mathbf{p}_{V T}=\mathbf{p}_{U}+l_{L T} \mathbf{e}_{\perp},
$$

where $l_{L T}$ is the constant distance, and $\mathbf{e}_{\perp}$ is calculated as follows: 


$$
\begin{aligned}
& \mathbf{e}_{\perp}=\mathbf{M}_{R} \mathbf{M}_{H} \mathbf{e}_{L O S}, \quad \mathbf{M}_{H}=\left[\begin{array}{lll}
1 & 0 & 0 \\
0 & 1 & 0 \\
0 & 0 & 0
\end{array}\right], \\
& \mathbf{M}_{R}=\left[\begin{array}{ccc}
\cos \left(90^{\circ}\right) & -\sin \left(90^{\circ}\right) & 0 \\
\sin \left(90^{\circ}\right) & \cos \left(90^{\circ}\right) & 0 \\
0 & 0 & 1
\end{array}\right] .
\end{aligned}
$$

\subsection{Target reacquisition}

In the TR mode, UAVs perform a local search to find the lost target. For this purpose, UAVs utilize the following guidance process: UAV continues to use Eq. (14) for a time duration $t_{\mathrm{c}, \mathrm{TR}}$. Then, it moves with no guidance for a time duration $t_{e}$ to increase the radius of its circular motion. This process is repeated until $t_{T R}$ is expired or the target signal is received again, the case in which the UAV returns from TR to AT mode (Figure 10(a)). Otherwise, the operating mode returns from TR to GS (Figure 10(b)).

\subsection{Approach fuel tanker}

At the beginning of this mode, the UAV approaches the fuel tanker using the approximate information, obtained from the database. However, as soon as the tanker is observed, for example using a visual seeker, the UAV approaches the fuel tanker using seeker measurements and joins the tanker.

To perform AFT efficiently, the UAV estimates the future position of fuel tanker according to the available information, obtained from the database. Thus, the UAV uses Eq. (16) to determine the estimated time of approach to the fuel tanker $\left(t_{a}\right)$.

$$
t_{a}=\frac{\left\|\mathbf{p}_{F T}-\mathbf{p}_{U}\right\|}{\mathbf{v}_{U}}
$$

where $\mathbf{p}_{F T}$ is the position vector of the fuel tanker. According to Figure 8 and Eq. (16), the position of fuel tanker at the end of AFT ( $\left.y_{F T, E A F T}\right)$ is estimated as follows:

$$
y_{F T, E A F T}=\left\{\begin{array}{lll}
0 & \text { if } & v_{F T} t_{a}+y_{F T}<0 \\
v_{F T} t_{a}+y_{F T} & \text { if } & 0 \leq v_{F T} t_{a}+y_{F T} \leq c \\
c & \text { if } & v_{F T} t_{a}+y_{F T}>c \quad(17)
\end{array}\right.
$$

where $v_{F T}$ is the velocity of the fuel tanker. Therefore,

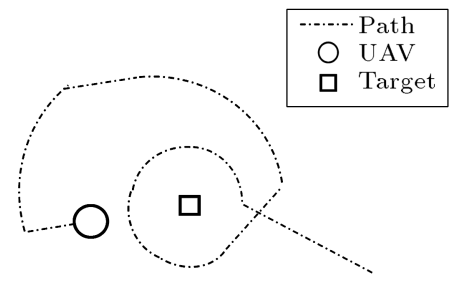

(a)

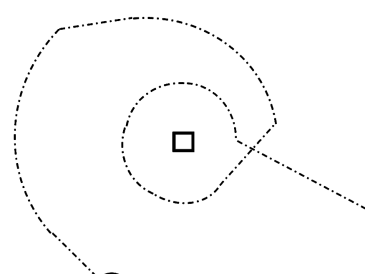

(b)
Figure 10. Target reacquisition: (a) Signal is received again, and (b) signal is lost completely. the UAV goes toward the future position of tanker instead of its current position. The UAV takes this estimated position as a virtual target and goes toward it.

When the distance between the UAV and fuel tanker becomes less than a certain value, the prediction stops and the position of the fuel tanker, obtained from the database, is used.

\subsection{Fueling}

When the UAV becomes close enough to the fuel tanker, it can perform its final approach using seeker information. In this mode, the target is the fuel tanker and the UAV is guided toward it. Moreover, an axial acceleration is applied to control the distance between the UAV and the fuel tanker $(d)$. This acceleration is a function of $d$ and its first derivative $(\dot{d})$.

$$
a_{x, c}=d \omega_{n}^{2}+2 \xi \omega_{n} \dot{d}
$$

where $a_{x, c}$ is the acceleration command in $x$ direction. Moreover, $\xi$ and $\omega_{n}$ are the desired damping ratio and the natural frequency of the distance control loop, respectively.

\section{Target localization using EKF}

The detected targets are localized using EKF. In the following, the stochastic dynamic model in the state space is utilized to define the target localization problem as a nonlinear states estimation problem. Then, the observability of the problem is analyzed. Finally, the target position is estimated using EKF.

\subsection{Formulation of the estimation problem}

According to Eq. (1), the relative motion equations of a non-maneuvering target with respect to a UAV are represented as follows:

$$
\begin{aligned}
\ddot{x}_{T}-\ddot{x}_{U}= & -a_{x, U} \cos \theta_{U} \cos \psi_{U}+a_{z, U} \\
& \left(\cos \phi_{U} \sin \theta_{U} \cos \psi_{U}-\sin \phi_{U} \sin \psi_{U}\right), \\
\ddot{y}_{T}-\ddot{y}_{U}= & -a_{x, U} \cos \theta_{U} \sin \psi_{U}+a_{z, U} \\
& \left(\cos \phi_{U} \sin \theta_{U} \sin \psi_{U}+\sin \phi_{U} \cos \psi_{U}\right) .
\end{aligned}
$$

Since there are always model uncertainties, measurement noises, and environmental disturbances, a stochastic model is required. Therefore, Eqs. (19) and (20) are augmented by process noises. By defining:

$$
\begin{aligned}
& x_{1}=x_{r}=x_{T}-x_{U}, \quad x_{2}=\dot{x}_{T} \dot{x}_{U}, \\
& x_{3}=y_{r}=y_{T}-y_{U}, \quad x_{4}=\dot{y}_{T}-\dot{y}_{U},
\end{aligned}
$$

the stochastic model can be expressed as follows: 


$$
\begin{aligned}
& \dot{x}_{1}=x_{2}+w_{1}, \\
& \dot{x}_{2}=-a_{x, U} \cos \theta_{U} \cos \psi_{U}+a_{z, U}
\end{aligned}
$$

$\left(\cos \phi_{U} \sin \theta_{U} \cos \psi_{U}-\sin \phi_{U} \sin \psi_{U}\right)+w_{2}$,

$$
\begin{aligned}
& \dot{x}_{3}=x_{4}+w_{3}, \\
& \dot{x}_{4}=-a_{x, U} \cos \theta_{U} \sin \psi_{U}+a_{z, U}
\end{aligned}
$$$$
\left(\cos \phi_{U} \sin \theta_{U} \sin \psi_{U}+\sin \phi_{U} \cos \psi_{U}\right)+w_{4},
$$

where $w_{i}(i=1 \ldots 4)$ are zero mean white noises with known covariance, and $\mathbf{Q}$ represents the process noise. These equations are used in EKF to estimate the relative states. The state estimation is performed at discrete time; hence, the above equations should be discretized with sampling time $T_{s}$. After discretization, Eqs. (21)-(24) are written as follows:

$$
\begin{aligned}
& x_{1}(k)=x_{1}(k-1)+x_{2}(k-1) T_{s}+T_{s} w_{1}, \\
& x_{2}(k)=x_{2}(k-1)+T_{s}\left(-a_{x, U} \cos \theta_{U} \cos \psi_{U}+a_{z, U}\right.
\end{aligned}
$$

$$
\left(\cos \phi_{U} \sin \theta_{U} \cos \psi_{U}-\sin \phi_{U} \sin \psi_{U}\right)+T_{s} w_{2},
$$

$x_{3}(k)=x_{3}(k-1)+x_{4}(k-1) T_{s}+T_{s} w_{3}$,

$x_{4}(k)=x_{4}(k-1)+T_{s}\left(-a_{x, U} \cos \theta_{U} \sin \psi_{U}+a_{z, U}\right.$

$$
\left.\left(\cos \phi_{U} \sin \theta_{U} \sin \psi_{U}-\sin \phi_{U} \cos \psi_{U}\right)\right)+T_{s} w_{4}
$$

In addition, the outputs measured by the sensors of each UAV include azimuth angle $(\sigma)$ and the elevation angle $(\varepsilon)$ of the detected target. Therefore, according to Figure 11, the measurement model can be written as follows:

$$
\begin{aligned}
& y_{1}=\varepsilon+v_{1}=\tan ^{-1}\left(\frac{z_{r}}{x_{r}}\right)+v_{1}=\tan ^{-1}\left(\frac{\Delta H}{x_{1}}\right)+v_{1} \\
& y_{2}=\sigma+v_{2}=\tan ^{-1}\left(\frac{y_{r}}{x_{r}}\right)+v_{2}=\tan ^{-1}\left(\frac{x_{3}}{x_{1}}\right)+v_{2}
\end{aligned}
$$

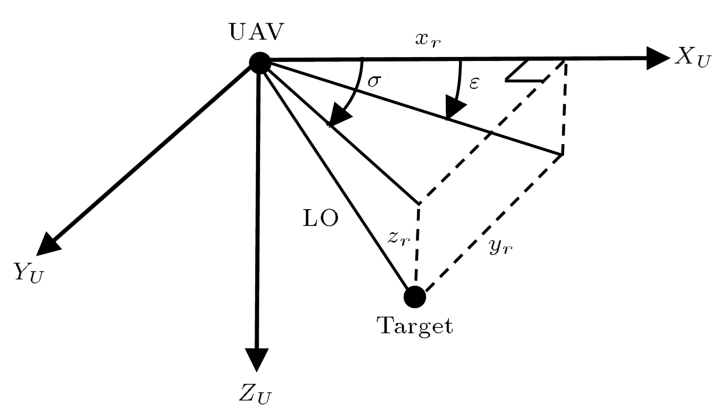

Figure 11. The azimuth and elevation angles of target measured by UAV. where $v_{1}$ and $v_{2}$ are zero mean white noises with known covariance. In addition, $z_{r}=z_{T}-z_{U}$ is equal to altitude difference $(\Delta H)$ during the state estimation.

\subsection{Observability analysis}

It is necessary to investigate the observability of the states from the available measurements. The conducted researches for observability analysis can be categorized to linear and nonlinear systems [36]. The observability of a linear system is investigated via the matrix rank calculations [37]. On the contrary, the observability of a nonlinear system [36-38] is examined via local linearization rank [36]. In this section, the observability of the relative states $\left(x_{r}\right.$ and $\left.y_{r}\right)$ is analyzed using the theory of nonlinear systems observability. The state space representation of a nonlinear system is assumed to be modeled as follows [36]:

$$
\dot{\mathbf{x}}=\mathbf{f}(\mathbf{x}, \mathbf{u})
$$

where elements of $\mathbf{f}$ are nonlinear functions of the state vector, $\mathbf{x}$, and the input vector, u. Moreover, the measurements are related to the states via the measurement equation stated as follows:

$$
\mathbf{y}=\mathbf{h}(\mathbf{x})=\left[h_{1}(\mathbf{x}), \ldots \ldots \ldots \ldots . . . . h_{p}(\mathbf{x})\right]^{T},
$$

where elements of $h(\mathbf{x})$ are nonlinear functions, too. The mathematical calculations of the observability analysis are presented in the Appendix.

\subsection{Extended Kalman filter}

In this paper, since the measurement models are nonlinear, EKF [39] is utilized to estimate the relative states. EKF consists of two essentially stages: prediction and update [40]. Prediction uses the estimated states of the previous time step to produce a priori estimate of the current step. In this step, the estimated states and its covariance matrix are expressed as follows:

$$
\begin{aligned}
& \hat{\mathbf{x}}_{k \mid k-1}=\mathbf{f}\left(\hat{\mathbf{x}}_{k-1 \mid k-1}\right), \\
& \mathbf{P}_{k \mid k-1}=\mathbf{Q}+\mathbf{F}_{k-1} \mathbf{P}_{k-1 \mid k-1} \mathbf{F}_{k-1}^{T},
\end{aligned}
$$

where $\mathbf{F}_{k-1}$ is the Jacobian matrix of nonlinear functions $\mathbf{f}_{k-1}$ obtained as follows:

$$
\begin{aligned}
\mathbf{F} & =\left[\nabla_{\mathbf{x}(k-1)} \mathbf{f}^{T}(\mathbf{x}(k-1))\right]_{\mathbf{X}_{k-1}=\hat{\mathbf{x}}_{k-1 \mid k-1}}^{T} \\
& =\left[\begin{array}{cccc}
1 & T_{s} & 0 & 0 \\
0 & 1 & 0 & 0 \\
0 & 0 & 1 & T_{s} \\
0 & 0 & 0 & 1
\end{array}\right]^{T} .
\end{aligned}
$$

In the update stage, the priori state estimation is combined with the current observation to refine 
the state estimation and obtain the posterior state estimation. In this step, the estimated states and the corresponding covariance matrix are expressed as follows:

$$
\begin{aligned}
& \hat{\mathbf{x}}_{k \mid k}=\hat{\mathbf{x}}_{k \mid k-1}+\mathbf{K}_{k}\left(\mathbf{z}_{k}-\mathbf{h}\left(\hat{\mathbf{x}}_{k \mid k-1}\right)\right), \\
& \mathbf{P}_{k \mid k}=\mathbf{P}_{k \mid k-1}-\mathbf{K}_{k}\left(\mathbf{H}_{k} \mathbf{P}_{k \mid k-1} \mathbf{H}_{k}^{T}+\mathbf{R}\right) \mathbf{K}_{k}^{T},
\end{aligned}
$$

where $\mathbf{K}_{k}$ is the Kalman gain matrix calculated as follows:

$$
\mathbf{K}_{k}=\mathbf{P}_{k \mid k-1} \mathbf{H}_{k}^{T}\left(\mathbf{H}_{k} \mathbf{P}_{k \mid k-1} \mathbf{H}_{k}^{T}+\mathbf{R}\right)^{-1},
$$

where $\mathbf{H}_{k}$ is the Jacobian matrix of nonlinear functions $\mathbf{h}_{k}$ obtained as follows:

$$
\begin{aligned}
\mathbf{H}_{k} & =\left[\nabla_{\mathbf{x}(\mathbf{k})} \mathbf{h}^{T}(\mathbf{x}(k))\right]_{\mathbf{x}_{k}=\hat{\mathbf{x}}_{k \mid k-1}}^{T} \\
& =\left[\begin{array}{cccc}
-\frac{\Delta H}{\Delta H^{2}+x_{1}^{2}} & 0 & 0 & 0 \\
-\frac{x_{3}}{x_{1}^{2}+x_{3}^{2}} & 0 & \frac{x_{1}}{x_{1}^{2}+x_{3}^{2}} & 0
\end{array}\right] .
\end{aligned}
$$

Therefore, the relative states are estimated through Eq. (36), and target states are obtained as:

$$
\begin{aligned}
& \hat{x}_{T}=x_{U}+\hat{x}_{1}, \\
& \hat{v}_{x_{T}}=\hat{x}_{2}+v_{x_{U}}, \\
& \hat{y}_{T}=\hat{x}_{3}+y_{U}, \\
& \hat{v}_{y_{T}}=\hat{x}_{4}+v_{y_{U}} .
\end{aligned}
$$

\section{Simulation results}

In this section, simulation results are presented. Assume that three UAVs cooperatively search a region

\begin{tabular}{|c|c|c|}
\hline Parameters & Values & Unit \\
\hline $\mathbf{R}$ & $(1 e-4) \operatorname{diag}(1,1)$ & - \\
\hline $\mathbf{P}_{0}$ & $100 \operatorname{diag}(100,1,100,1)$ & - \\
\hline $\mathbf{Q}$ & $(1 e-5) \operatorname{diag}(5,6,7,8)$ & - \\
\hline$f_{\max }$ & 100 & $\mathrm{~kg}$ \\
\hline $\mathrm{r}_{F U}$ & 0.03 & $\mathrm{~kg} / \mathrm{sec}$ \\
\hline $\mathrm{b}$ & 100 & $\mathrm{~km}$ \\
\hline$t_{f u}$ & 120 & $\mathrm{sec}$ \\
\hline c & 100 & $\mathrm{~km}$ \\
\hline$T_{s}$ & 0.02 & $\mathrm{sec}$ \\
\hline$n_{x}$ & 4 & - \\
\hline$n_{y}$ & 4 & - \\
\hline$k_{V P}$ & 15 & - \\
\hline$l_{L T}$ & 30 & $\mathrm{~m}$ \\
\hline$l_{A T}$ & 100 & $\mathrm{~m}$ \\
\hline$v_{F T}$ & 100 & $\mathrm{~m} / \mathrm{s}$ \\
\hline$t_{u}$ & 5 & $\mathrm{sec}$ \\
\hline$t_{e}$ & 30 & $\mathrm{sec}$ \\
\hline$t_{T R}$ & 360 & $\mathrm{sec}$ \\
\hline$g$ & 10 & $\mathrm{~m} / \mathrm{s}^{2}$ \\
\hline$\xi$ & 0.9 & - \\
\hline$\omega_{n}$ & 0.3 & $\mathrm{rad} / \mathrm{sec}$ \\
\hline
\end{tabular}
of the size $100 \times 100 \mathrm{~km}^{2}$ and localize five mobile ground targets. UAVs take fuel from an air fuel tanker.

\begin{tabular}{|c|c|c|c|c|c|c|c|c|c|}
\hline \multicolumn{2}{|c|}{ Vehicles } & \multicolumn{3}{|c|}{$(\mathrm{km})$} & $V_{X 0}$ & \multicolumn{2}{|c|}{$(\mathrm{m} / \mathrm{s})$} & ${ }_{(\mathrm{ra}}^{\psi_{\mathrm{o}}}$ & $\left.\theta_{0}\right]$ \\
\hline \multirow{3}{*}{ UAVs } & 1 & {$[5$} & 20 & $5.05]$ & {$[100$} & $0 \quad c$ & & {$[0$} & 0] \\
\hline & 2 & {$[5$} & 45 & $5.1]$ & {$[100$} & 0 & & {$[0$} & $0]$ \\
\hline & 3 & {$[5$} & 75 & $5.15]$ & {$[100$} & 0 & & {$[0$} & 0] \\
\hline \multirow{5}{*}{ Targets } & 1 & {$[40$} & 15 & 0] & {$[0$} & -1 & & - & \\
\hline & 2 & {$[2.5$} & 1 & $5.05]$ & {$[0$} & $\left.\begin{array}{ll}10 & 0\end{array}\right]$ & & - & \\
\hline & 3 & {$[65$} & 90 & 0] & {$[-10$} & -2 & 0] & - & \\
\hline & 4 & {$[95$} & 40 & 0] & {$[-4$} & $4 \quad 0$ & & - & \\
\hline & 5 & {$[2$} & 95 & $5]$ & {$[10$} & -10 & 0] & - & \\
\hline \multicolumn{2}{|c|}{ Fuel tanker } & {$[0.3$} & 95 & $5]$ & {$[0$} & -100 & $0]$ & {$[3 \pi / 2$} & 0] \\
\hline
\end{tabular}
Initial conditions of the vehicles and the parameters of the cooperative search and localization are given in Tables 1 and 2, respectively. Moreover, the decision functions, utilized in global search and turn taking for

Table 2. Parameters of the cooperative search and localization algorithm.

Table 1. Initial conditions of UAVs, targets, and fuel tanker (km). 
fueling, are set as follows:

$$
\begin{aligned}
& f_{s}=1000 n_{s}+10 \frac{1}{t_{s}}+0.1 \frac{1}{\bar{d}}+100\left|\Delta \varphi_{U}\right| \\
& f_{T T}=p_{F}-0.01\left(\frac{n}{n_{U}-1}\right)-0.01\left(\frac{d_{F T}}{d_{F T, \max }}\right) .
\end{aligned}
$$

Figure 12 shows two-dimensional trajectory of the UAVs, targets, and fuel tanker. Generally, it can be observed that UAVs successfully search and locate the ground targets and take fuel from the fuel tanker whenever required.

In Figure 12(a), the first UAV (UAV1) receives a signal from the first target (T1) and approaches it. However, since the target signal has been lost during the LT mode, UAV1 goes to the TR mode in which the radius of the circular motion is increased. In addition, UAV3 receives a signal from T3 and approaches T3. In Figure 12(b), UAV1 is in the TR mode when a signal is received again from $\mathrm{T} 1$. Therefore, UAV1 approaches $\mathrm{T} 1$, localizes it, and then returns to the GS mode. Further, UAV3 localizes T3 and comes back to the GS mode. In Figure 12(c), all three UAVs are searching for the targets. In Figure 12(d), UAV1 receives a signal from T4, approaches it, determines its location, and returns to the GS mode. In Figure 12(e), all UAVs are in the GS mode and, then, switch to the AFT mode. In Figure 12(f), UAV1 is in the FUE mode, and UAV2 and UAV3 follow the fuel tanker. In Figure $12(\mathrm{~g})$, all UAVs have completed their fueling process. UAV2 approaches T2 and localizes it immediately after fueling and, then, goes to the GS mode. In the same time, UAV1 and UAV3 are in the GS mode. In Figure $12(\mathrm{~h})$ and (i), all UAVs are in the GS mode. In Figure 12(j), UAV3 receives a signal from $\mathrm{T} 5$, approaches $\mathrm{T} 5$, and localizes it.

Figure 13 shows the variation of fuel masses. The fuel percentage varies from 100 to $10 \%$; each UAV takes fuel at least one time, and the starting time of fueling is approximately 3000, 3200, and 3400 for UAV1, UAV2, and UAV3, respectively.

Figure 14 shows the accelerations and roll angle applied to UAV1. The longitudinal and normal accelerations of the body frame $\left(a_{x}\right.$ and $\left.a_{z}\right)$ are limited within the range $\left[\begin{array}{ll}-10 & 10\end{array}\right]$ and $\left[\begin{array}{ll}-15 & 15\end{array}\right], \mathrm{m} / \mathrm{s}^{2}$, respectively. In addition, the commanded roll angle $(\varphi)$ is limited within the range $\left[\begin{array}{ll}-60 & 60\end{array}\right] \mathrm{deg}$. To show the acceptable behavior of $a_{z}$ and $\varphi$, Figure 15 plots

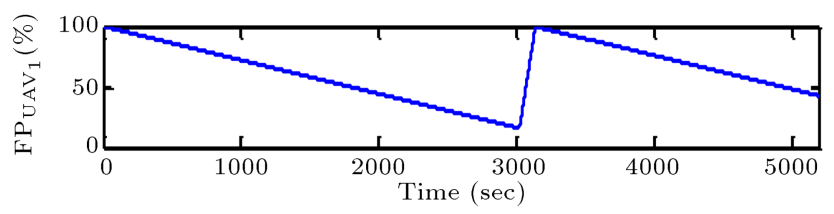

(a)

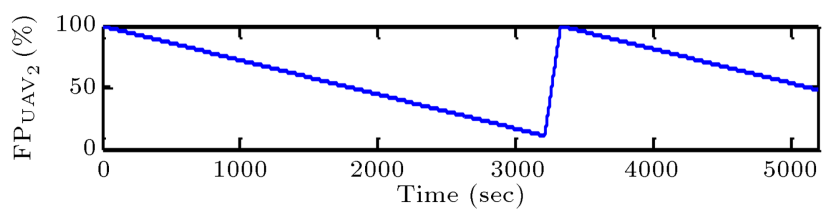

(b)

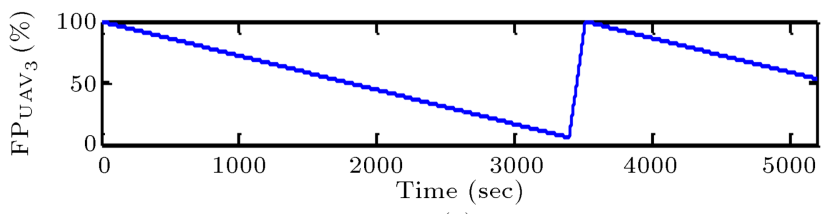

(c)

Figure 13. Fuel percentage of UAVs versus time: (a) UAV1, (b) UAV2, and (c) UAV3.

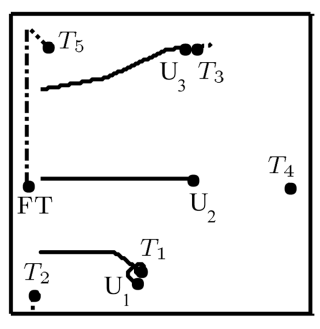

(a)

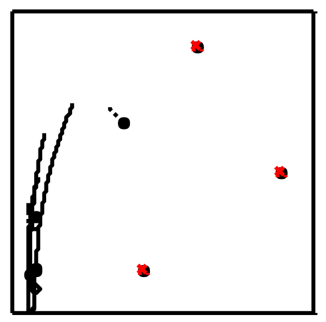

(f)

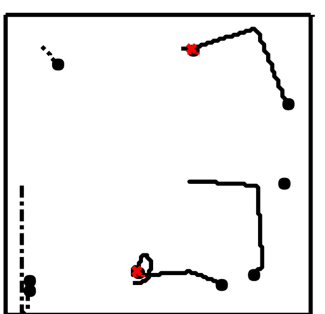

(b)

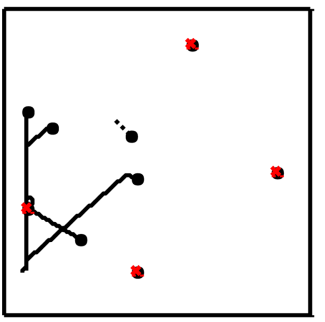

(g)

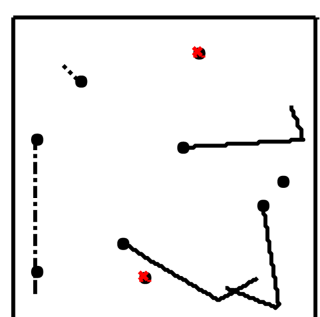

(c)

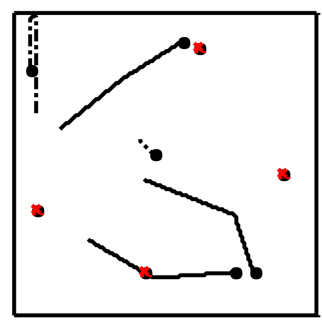

(h)

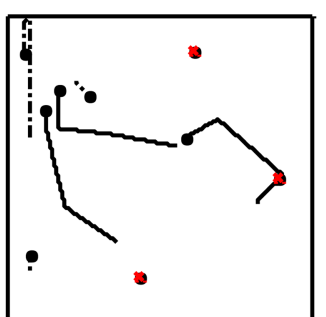

(d)

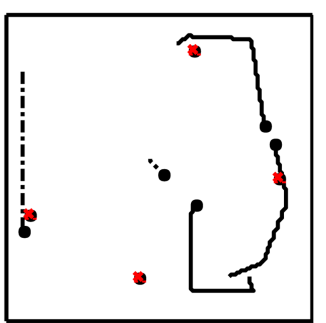

(i)

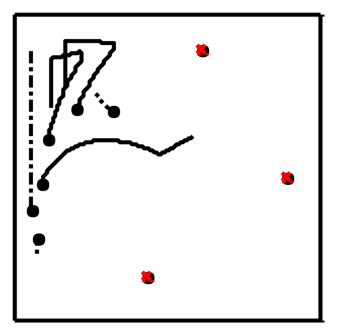

(e)

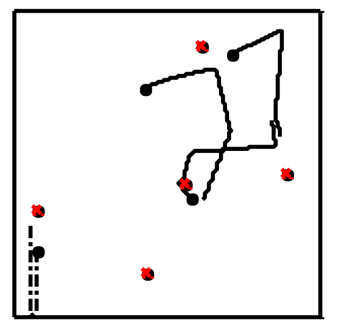

(j)

Figure 12. Snapshots showing the movement of UAVs (solid lines), targets (dotted lines), and fuel tanker (dash-dot lines). 


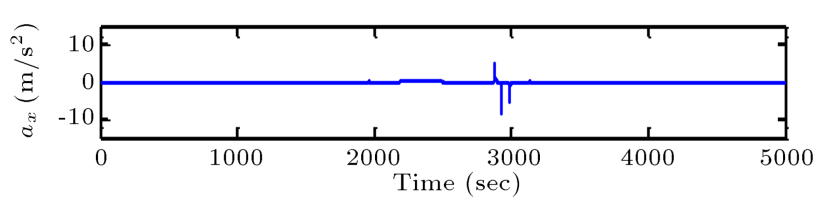

(a)

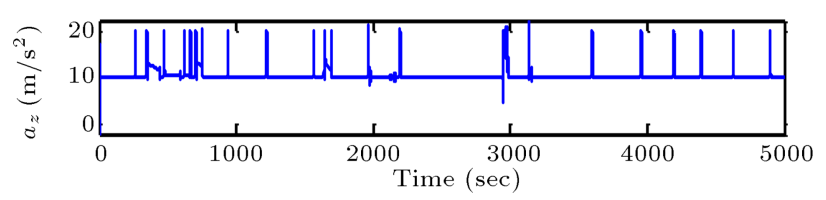

(b)

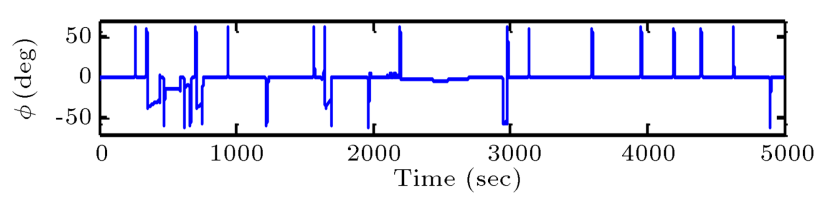

(c)

Figure 14. Accelerations and roll command applied to UAV1.

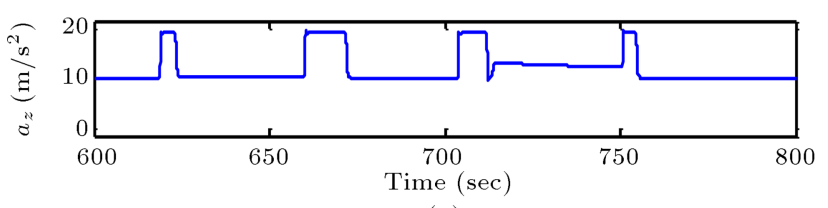

(a)

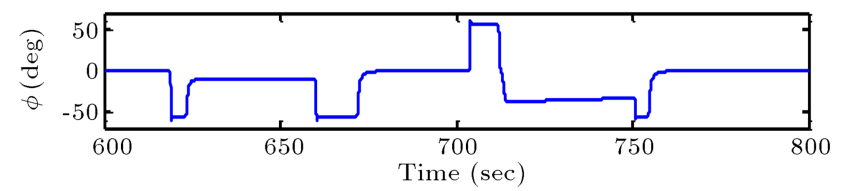

(b)

Figure 15. Normal acceleration and roll command applied to UAV1 between $t=600$ and $800 \mathrm{sec}$.

the variation of these commands in a narrow duration of flight.

Figure 16(a) shows the distance of UAV3 from the fuel tanker during the flight. Figure 16(b) shows that, at time intervals $[-3200330]$ [3200,3330], UAV3 flights at a distance of $970 \mathrm{~m}$ behind the fuel tanker until the fueling process of UAV2 is finished. Figure 16(c) shows that UAV3 keeps a constant distance from the fuel tanker during the fueling period.

Figure 17(a) shows the trajectory of UAV and target during the localization process. UAV receives a signal from the moving target, goes to the AT mode, performs a circular motion around the target, locates it, and returns to the global search mode. The indicators, plotted in Figure 17(b)-(d), show the time durations in which the operating modes are active.

Figure 18(a) and (b) presents the convergence of the estimated position of the target to a true value. When the AT mode starts at $t=30 \mathrm{sec}$, the estimated position starts to converge, too; after $t=100 \mathrm{sec}$, the convergence is completed. Figure 18(c) and (d) shows

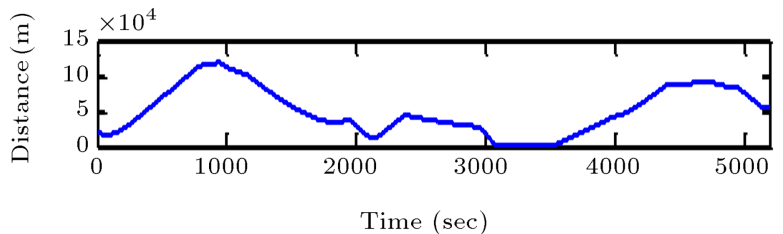

(a)

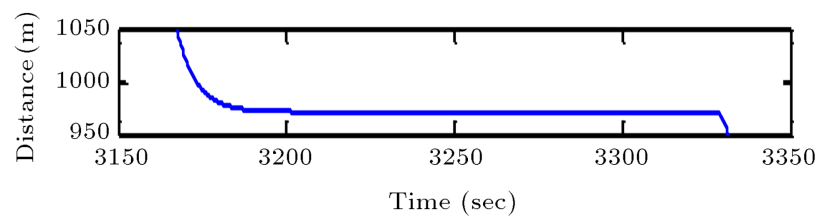

(b)

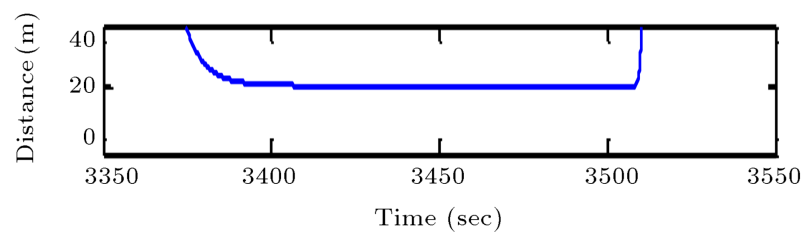

(c)

Figure 16. Distance of UAV3 from FT: (a) All flight, (b) waiting phase, and (c) fueling phase.

that the error in position is less than $10 \mathrm{~m}$ after $t=$ 120 sec. Figure 19 presents the covariance plots of the estimated states. All elements of the covariance matrix properly converge to zero.

In Figure 20, successful target reacquisition is investigated. According to Figure 20(a), during the LT mode, the target signal is lost; therefore, the UAV increases the radius of its circular motion in two steps in order to detect the target. Since the target is detected again before the maximum permitted TR time duration (320 seconds), the UAV approaches the target and locates it.

According to Figure 20(b), (c), and (d), the UAV is in the GS mode during the first 110 seconds of the flight; then, it goes to the AT and LT modes. Figure 20(e) shows that the signal is lost at $t=$ $110 \mathrm{sec}$, and the UAV goes to the TR mode. The target is detected again at $t=420$ sec; therefore, the UAV mode is switched to AT (Figure 20(c)). The target localization process begins at $t=420 \mathrm{sec}$ (Figure 20(d)). Finally, the UAV goes to the GS mode at $t=510 \mathrm{sec}$ (Figure 20(b)).

In Figure 21, a complete signal loss is investigated. Figure 21(a) shows that the target signal is lost during the LT mode; hence, the UAV mode changes to TR. Since the lost target is not detected before the maximum permitted TR time duration, the operating mode is switched to GS.

According to Figure 21(b), (c), and (d), the UAV is in the GS mode during the first 65 seconds of the flight; then, it goes to AT and LT modes. Figure 21(e) shows that the target signal is lost at $t=65 \mathrm{sec}$, and 


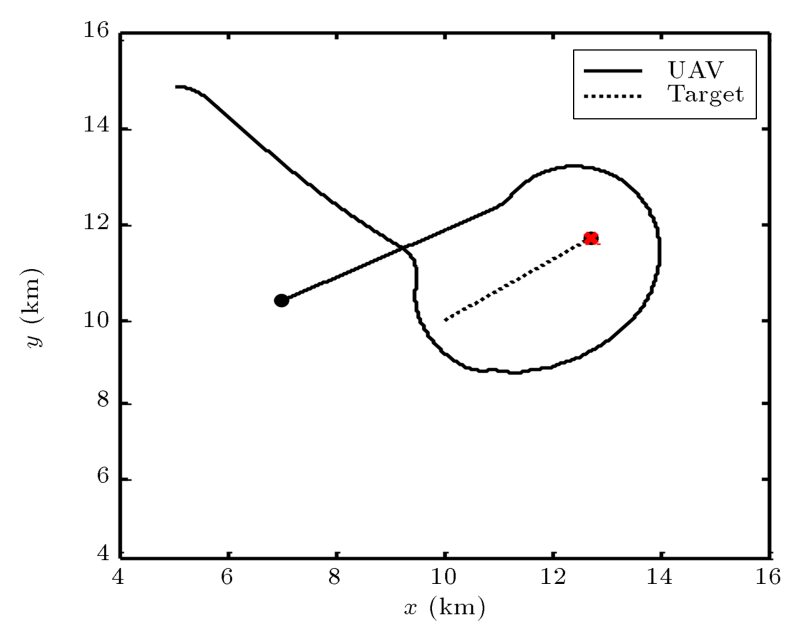

(a)

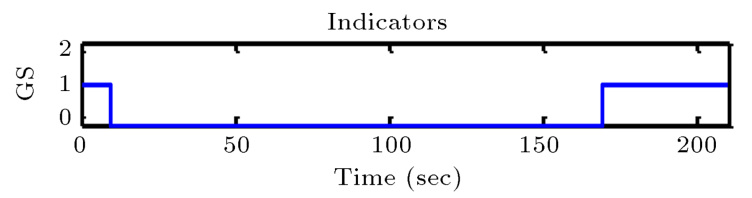

(b)

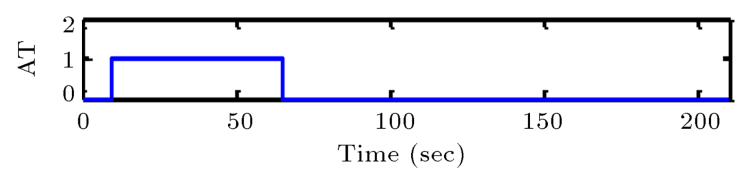

(c)

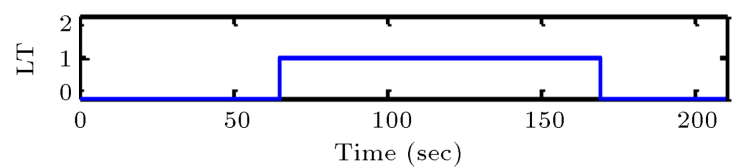

(d)

Figure 17. Search and localization of a moving target using a UAV: (a) 2-D paths of UAV and target, (b) GS indicator versus time, (c) AT indicator, and (d) LT indicator.

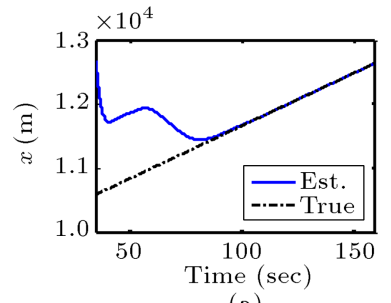

(a)

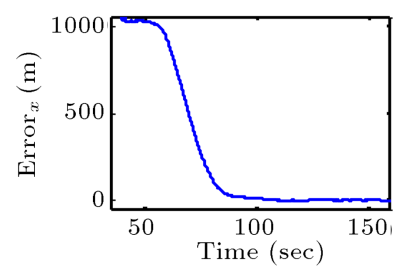

(c)

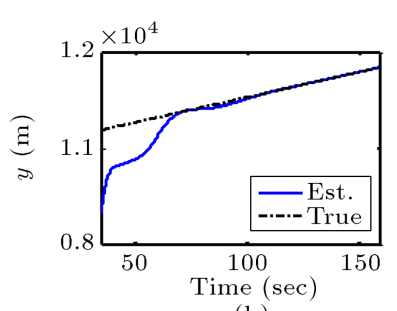

(b)

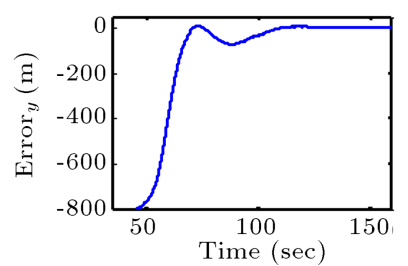

(d)
Figure 18. Estimation of $x_{T}$ and $y_{T}$ : (a) Convergence of the estimated $x_{T}$ to true value, (b) convergence of the estimated $y_{T},(\mathrm{c})$ estimation error of $x_{T}$, and (d) estimation error of $y_{T}$.

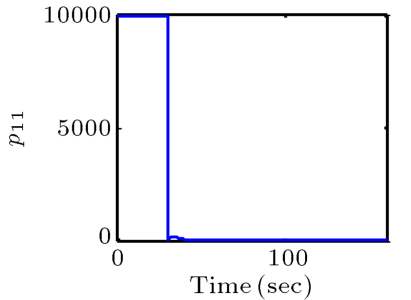

(a)

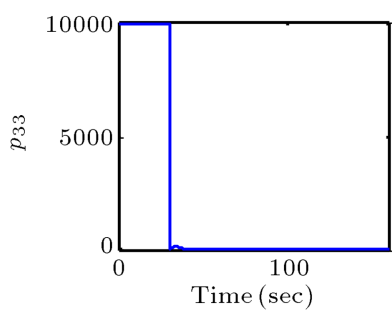

(c)

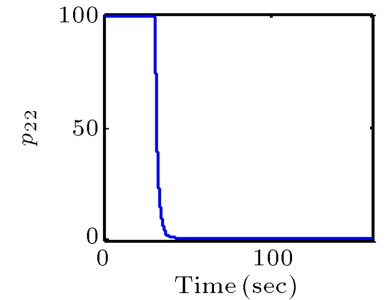

(b)

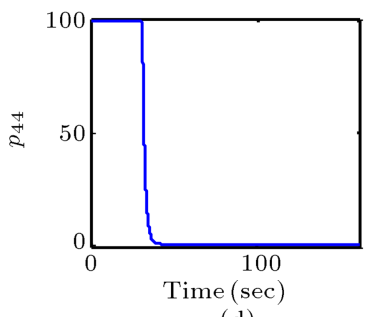

(d)
Figure 19. Elements of the covariance matrix of the estimated states.

the UAV mode is switched to TR. Since the TR time expires, the UAV mode is switched back to GS at $t=$ $465 \mathrm{sec}$ (Figure 21(b)).

In the following, a scenario for investigating the AFT and FUE modes of a UAV is presented. The UAV utilizes the decision function of Eq. (45) to take turns. In addition, it is considered that $f_{T T, C}=0.99$; therefore, immediately after satisfying the condition $f_{T T} \leq 0.99$, the UAV takes turns for fueling and goes to the AFT mode. According to Figure 22(a), the condition $f_{T T} \leq 0.99$ is satisfied approximately at $t=90 \mathrm{sec}$. In addition, Figure 22(b) shows the variation in fuel percent. According to this figure, the start and end times of the FUE mode are 320 and 620 , respectively.

Figure 23(a) shows the UAV path during the GS, AFT, and FUE modes. According to Figure 23(b), the UAV is in the GS mode during the first 90 seconds of the flight. The UAV goes to the AFT mode at $t=90 \mathrm{sec}$ (Figure 23(c)). Then, the UAV is in the FUE mode between $t=320$ and $620 \mathrm{sec}$ (Figure 23(d)). When the fueling process ends at $t=620 \mathrm{sec}$, the UAV goes back to the GS mode (Figure 23(b)).

Since the UAV altitude differs from the FT altitude, the altitude of UAV changes from $5.05 \mathrm{~km}$ to $5 \mathrm{~km}$ during FUE process (Figure 24) and goes back to $5.05 \mathrm{~km}$ after the fueling process.

To investigate the performance of the cooperative search and localization algorithm in different conditions, a Mont Carlo simulation is performed. In this case, 100 scenarios with a random initial position and velocity for UAVs and targets are simulated. The initial position of UAVs is bounded within the range $[2,25] \mathrm{km}$ in the $x$ direction and $[2,98] \mathrm{km}$ in the $y$ direction. Moreover, the initial velocities of the UAVs in the $x$ and $y$ directions are $100 \mathrm{~m} / \mathrm{s}$ and 0 , 


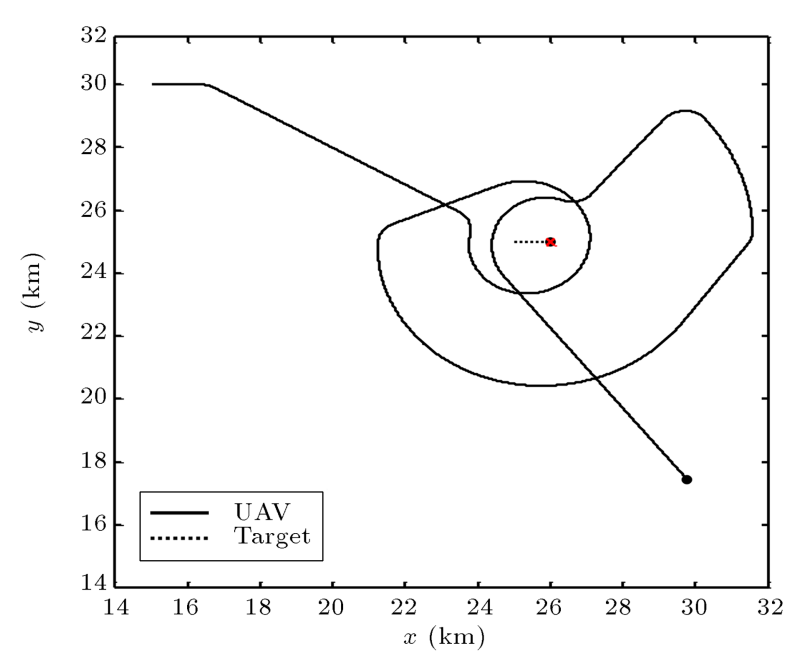

(a)

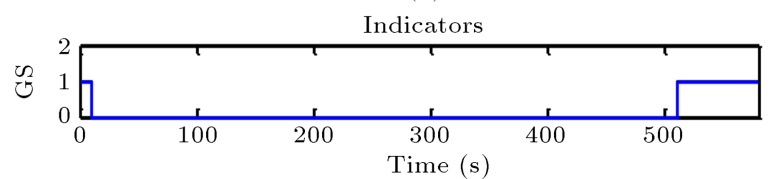

(b)

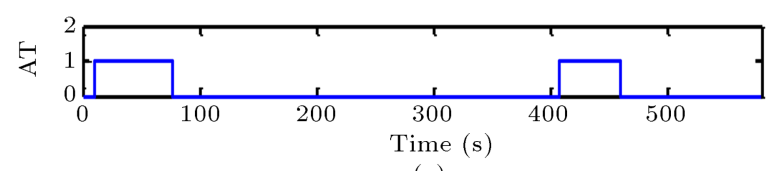

(c)

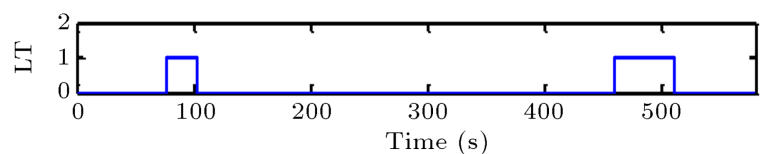

(d)

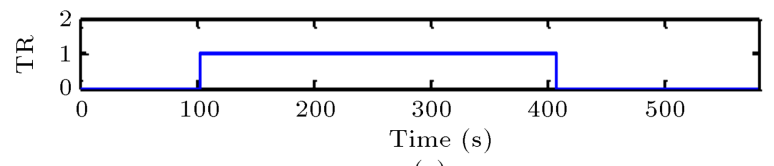

(e)

Figure 20. A sample flight in GS, AT, LT and TR modes: (a) 2D paths and positions of a UAV and a static target, (b) GS indicator versus time, (c) AT indicator, (d) LT indicator, and (e) TR indicator.

Table 3. The average performance of the algorithm obtained from Monte Carlo simulation.

\begin{tabular}{lcc}
\hline \multicolumn{1}{c}{ Parameter } & Value & Unit \\
\hline Success percentage & $\approx 95$ & $\%$ \\
Average search and localization time & 2688 & Sec \\
Average no. of located targets & 4.96 & - \\
Index of search uniformity & 2.55 & - \\
\hline
\end{tabular}

respectively. In addition, targets are located randomly with a random initial velocity within the range $[50$, 100] $\mathrm{km} / \mathrm{h}$ and a random initial heading within the range $[0,360]^{\circ}$. The simulation stops when all targets are located or $t>5000 \mathrm{sec}$.

Table 3 represents the average search and local-

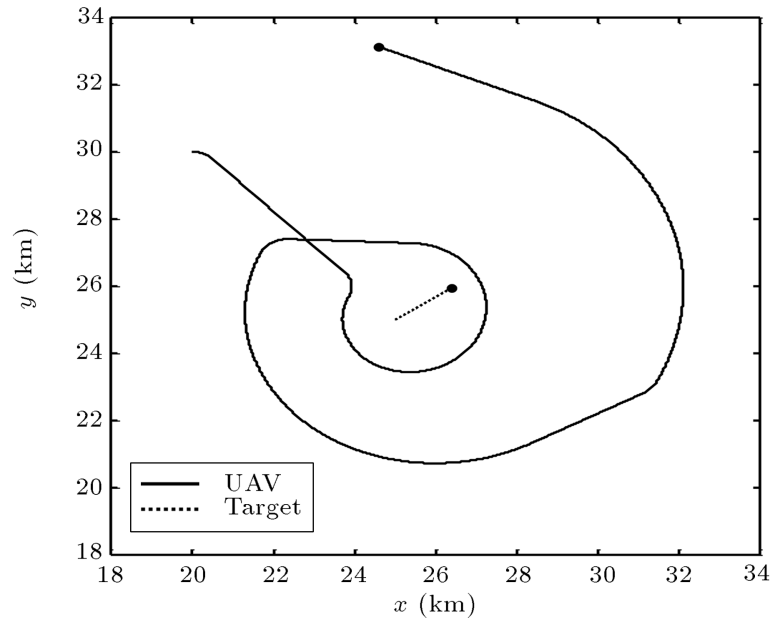

(a)

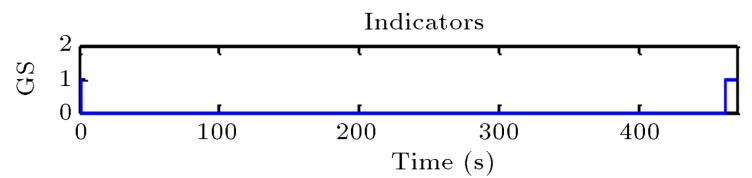

(b)

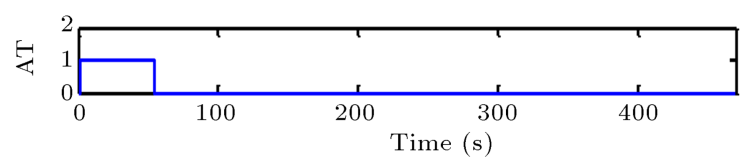

(c)

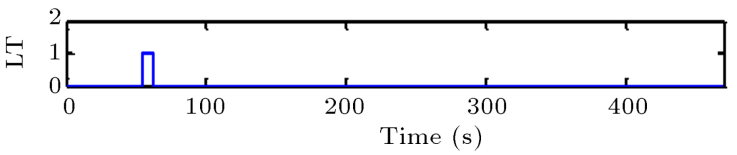

(d)

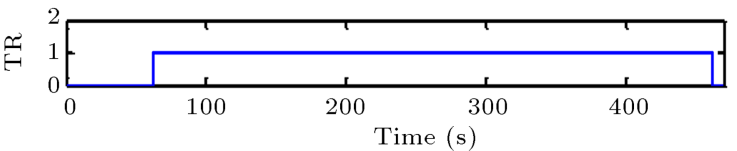

(e)

Figure 21. A condition of complete signal loss after LT mode: (a) 2-D paths of UAV and target, (b) GS indicator versus time, (c) AT indicator, (d) LT indicator, and (e) TR indicator.

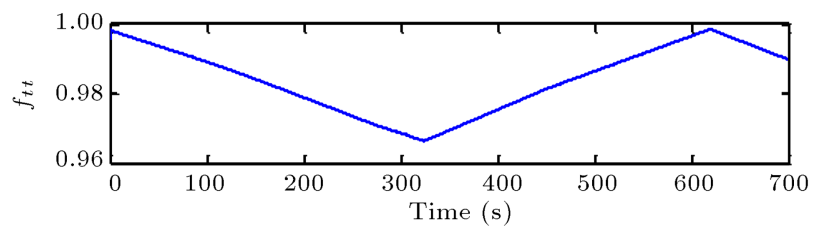

(a)

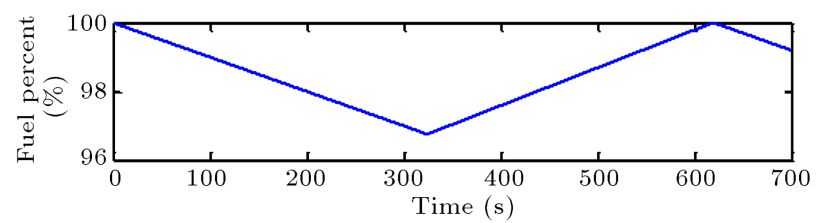

(b)

Figure 22. Variation of the turn-taking decision function and the fuel percent versus time. 


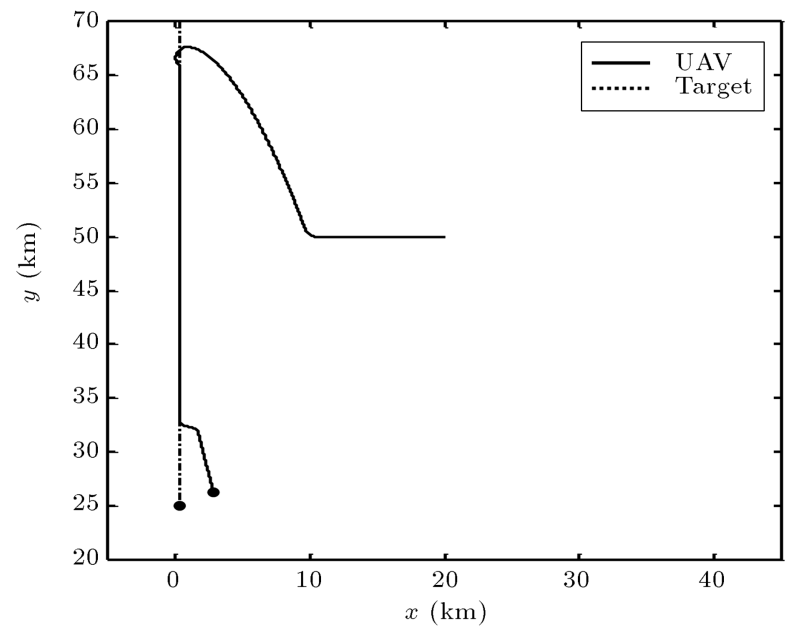

(a)

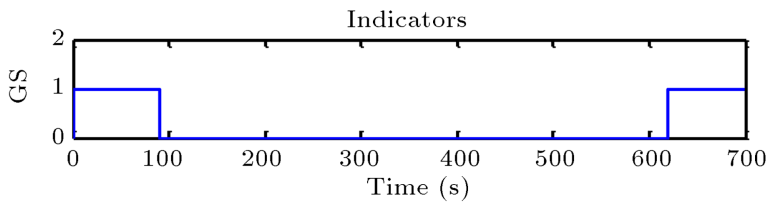

(b)

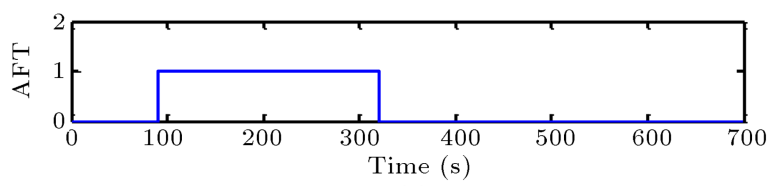

(c)

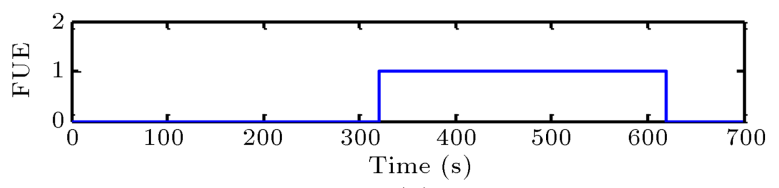

(d)

Figure 23. A sample flight in GS, AFT, and FUE modes: (a) 2-D paths of UAV and fuel tanker, (b) GS indicator versus time, (c) AFT indicator, and (d) FUE indicator.

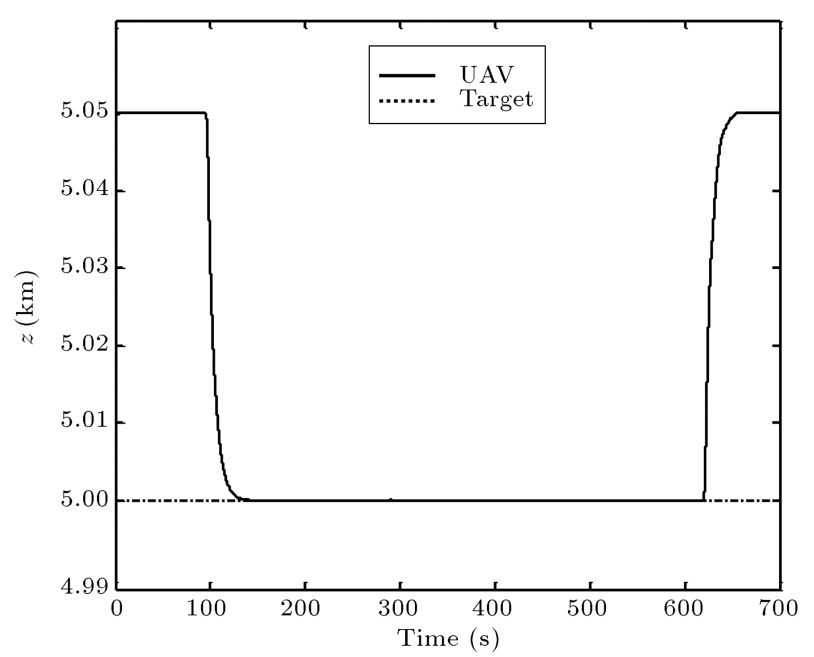

Figure 24. Variation of altitude during a sample flight in GS, AFT, and FUE modes.

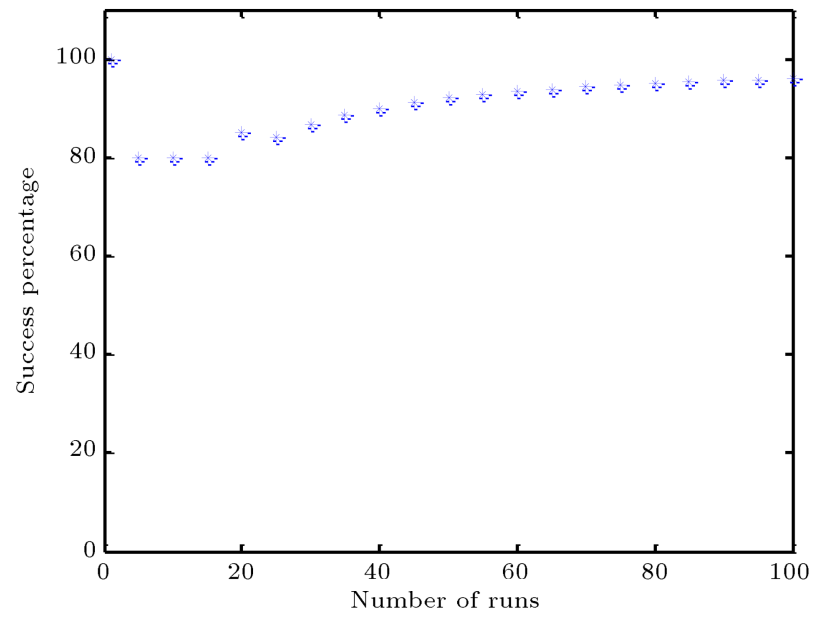

Figure 25. Success percentage versus the number of runs (from 1 to 100 runs).

ization time, the average number of located targets, success percent, and the average difference between the maximum and minimum seen number of zones that shows the uniformity of search. The success percentage indicates the success rate of UAVs in locating all targets before the final simulation time $(t=5000)$ is reached. The average search time is computed for successful runs. However, the average number of located targets and the search uniformity index are calculated for all 100 runs. The variation of the success percentage versus the number of runs is shown in Figure 25. This figure shows that 100 runs are sufficient for the convergence of the statistical properties. The success percentage is calculated as follows:

$$
S P=\frac{N S}{N R} \times 100,
$$

where $N R$ is the number of runs, $N S$ is the number of successful runs (the simulations in which all targets are located), and $S P$ is the success percentage.

To evaluate the proposed search and localization algorithm for a greater number of UAVs and targets, another scenario with 10 targets and 5 UAVs is investigated, the results of which are presented in Figure 26.

In Figure 26(a), UAV5 receives a signal from T7 and localizes it. In Figure 26(b), UAV5 localizes another target. In Figure 26(c), all UAVs are in the GS mode. In Figure 26(d), two other targets are localized. In Figure 26(e), another two targets are localized. In Figure 26(f), again, a new target is localized by one of the UAVs. In Figure 26(g), all UAVs are in the GS mode. In Figure 26(h), one of the UAVs starts to approach one of the targets. In Figure 26(i), one of the UAVs is taking fuel from the fuel tanker, another UAV is localizing one of the targets, and other UAVs are in the GS mode. In Figure 26(j), all targets are localized by the UAVs. It should be noted that, in this scenario, the total search and localization time is 2310 seconds. 


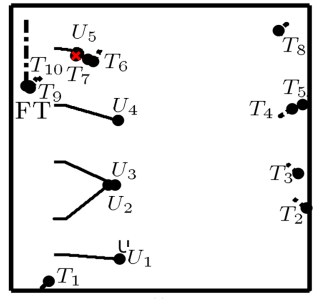

(a)

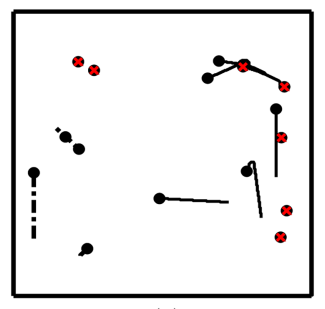

(f)

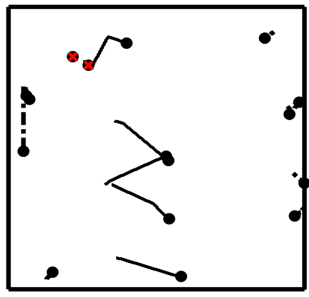

(b)

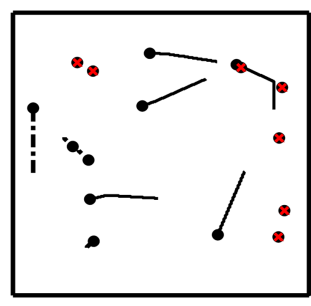

$(\mathrm{g})$

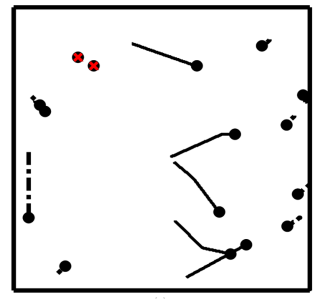

(c)

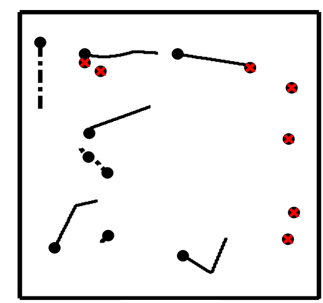

(h)

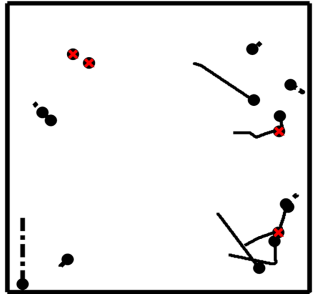

(d)

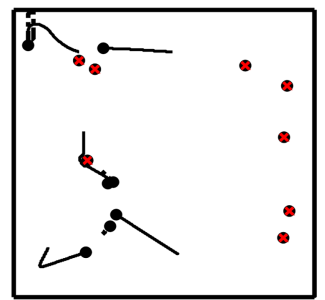

(i)

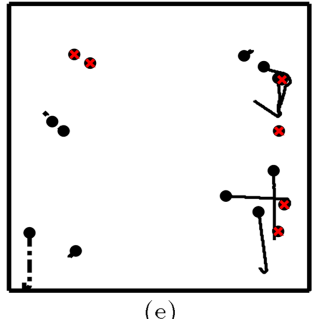

(e)

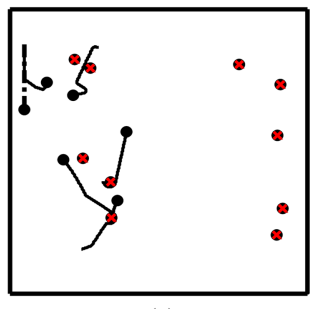

(j)

Figure 26. Snapshots showing the movement of UAVs (solid lines), targets (dotted lines), and fuel tanker (dash-dot line), 5 UAVs, and 10 targets.

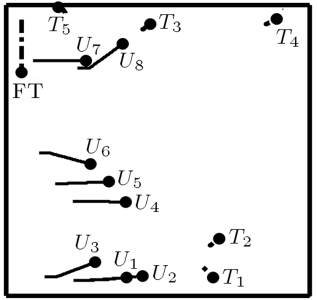

(a)

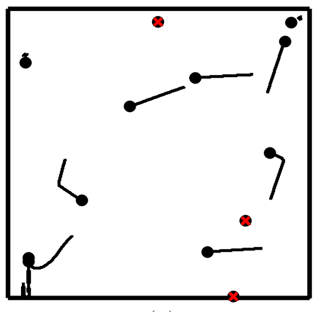

(f)

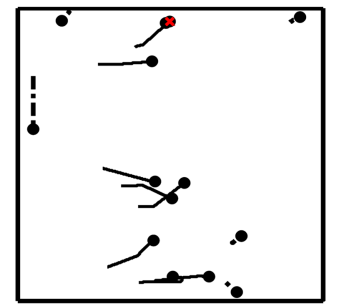

(b)

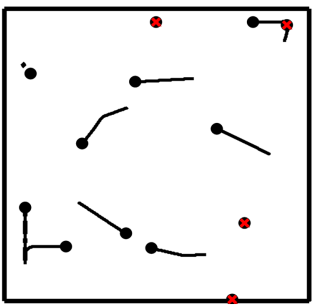

(g)

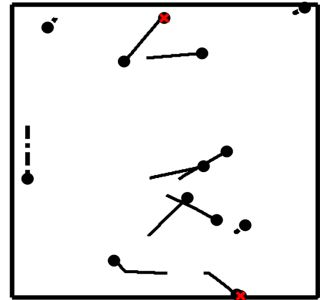

(c)

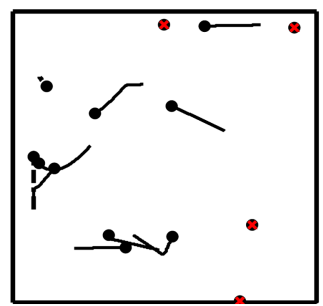

(h)

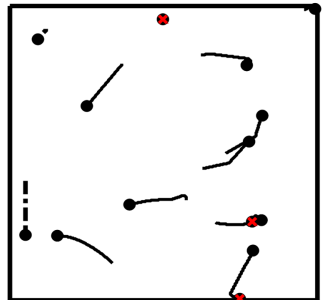

(d)

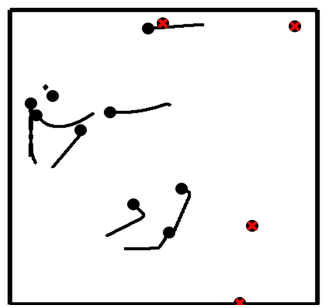

(i)

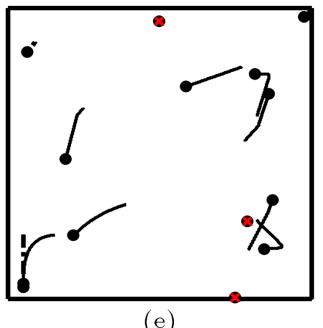

(e)

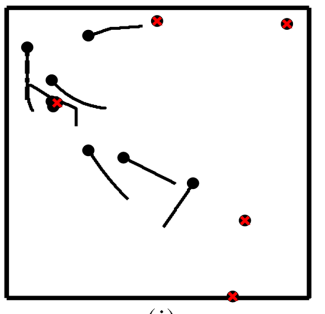

(j)

Figure 27. Snapshots showing the movement of UAVs (solid lines), targets (dotted lines), and fuel tanker (dash-dot line), 8 UAVs, and 5 targets.

In addition, to investigate the search and localization algorithm when the number of UAVs is greater than the number of targets, a scenario with 8 UAVs and 5 targets is simulated (Figure 27).

In Figure 27(a), all UAVs are in the GS mode. In Figure 27(b), one of the targets is localized. In Figure 27(c), a new target is localized. In Figure 27(d), again, another target is localized. In Figure 27(e) and (f), one of the UAVs is fueling from fuel tanker, one of the UAVs is approaching the fuel tanker, and other UAVs are in the GS mode. In Figure 27(g), a new target is localized. In Figure 27(h), some of the UAVs are in the GS mode, one of the UAVs is in the AFT mode, and another UAV is taking fuel from the fuel tanker. Finally, in Figure 27(i) and (j), the last target is localized, while some of the UAVs are in the AFT and FUE modes. The total search and localization time of this scenario is 1820 seconds.

\section{Conclusion}

In this article, cooperative search and localization of ground moving targets by a group of UAVs was investigated in the presence of fuel constraint. UAVs use six operating modes including GS, AT, LT, TR, AFT, and FUE. In the GS mode, the search area was divided into small zones, and a new decision function was proposed for UAVs to sequentially select their search zones such that all zones could be covered uniformly by the group of UAVs. In the LT mode, 
a new guidance algorithm was proposed to provide a near-circular motion of UAVs around the target, and EKF was used for target localization using the bearingonly measurements of the UAV. In the TR mode, a new idea was proposed to increase the radius of circular motion such that the UAV did not miss the chance of target reacquisition. In the AFT mode, a turn-taking decision function was proposed to manage the fueling process of the UAVs, serially. In addition, when several UAVs were in the AFT mode simultaneously, those UAVs that were not in the queue in the first place queued up behind the fuel tanker until their fueling time was reached.

The performance of the proposed algorithms was verified through numerical simulations. In addition, according to Monte Carlo simulations, in a $100 * 100 \mathrm{~km}$ search area, the success rate of $80 \%$ (at least) was obtained in the finding and localization of five random targets in less than $5000 \mathrm{sec}$. Moreover, the zone selection function shows a high performance to manage UAVs to visit all zones as uniformly as possible. In addition, it should be mentioned that there are some limitations for the proposed algorithm; removing these limitations can be considered in some future works. First, if the number of UAVs increased greatly, more than one fuel tanker would be required. Second, it was assumed that UAVs flew in different altitudes to avoid collision. However, for a great number of UAVs, there will be some limitations as to how to put different UAVs at different altitudes and how to manage them to change their altitude when they are switching to the AFT mode and switching back to the GS mode.

\section{Acknowledgements}

The authors gratefully acknowledge financial supports of the research office at Sharif University of Technology.

\section{References}

1. O'rourke, J. Art Gallery Theorems and Algorithms, 57, Oxford: Oxford University Press (1987).

2. Parker, L.E. and Emmons, B.A. "Cooperative multi-robot observation of multiple moving targets", Robotics and Automation, Proceedings, IEEE International Conference on, IEEE, 3 (1997).

3. Dehghan, S.M., Saberi, M., Tavakkoli, M., and Moradi, H. "Path planning for localization of an RF source by multiple UAVs on the Crammer-Rao lower bound", In Robotics and Mechatronics (ICRoM), First RSI/ISM International Conference on, IEEE, pp. 68-73 (2013).

4. Stegagno, P., Cognetti, M., Rosa, L., Peliti, P., and Oriolo, G. "Relative localization and identification in a heterogeneous multi-robot system", In: Robotics and Automation (ICRA), IEEE International Conference on, IEEE, pp. 1857-1864 (2013).
5. Natalizio, E., Surace, R., Loscrí, V., Guerriero, F., and Melodia, T. "Two families of algorithms to film sport events with flying robots", In IEEE 10th International Conference on Mobile Ad-Hoc and Sensor Systems, IEEE, pp. 319-323 (2013).

6. Chen, S., Fang, D., Chen, X., Xia, T., and Jin, M. "Aerial wireless localization using target-guided flight route", In ACM SIGCOMM Computer Communication Review ACM, p. 587-588 (2014).

7. Ferreira, S., Carvalho, G., Ferreira, F., and Sousa, J. "Assessing the capacity of man-portable UAVs for network access point localization, using RSSI link data", In Unmanned Aircraft Systems (ICUAS), International Conference on, IEEE, pp. 355-364 (2014).

8. Effati, M. and Krzysztof, S. "EKF and UKF localization of a moving $\mathrm{RF}$ ground target using a flying vehicle", IEEE 30th Canadian Conference on Electrical and Computer Engineering (CCECE), pp. 1-4 (2017).

9. Deghat, M., Xia, L., Anderson, B.D., and Hong, Y. "Multi-target localization and circumnavigation by a single agent using bearing measurements", International Journal of Robust and Nonlinear Control, 25(14), pp. 2362-2374 (2015).

10. Zhou, D., Zhang, H., Pan, Q., and Zhang, K. "An improved range parameterized square root cubature information filter algorithm for multi-UAV cooperative passive location", In Information and Automation, International Conference on, IEEE, pp. 1079-1084 (2015).

11. Nagaty, A., Thibault, C., Trentini, M., Facchinetti, T., and Li, H. "Construction, modeling and control of a quadrotor for target localization", In Electrical and Computer Engineering (CCECE), IEEE 28th Canadian Conference on, IEEE, pp. 308-313 (2015).

12. Hausman, K., Müller, J., Hariharan, A., Ayanian, N., and Sukhatme, G.S. "Cooperative multi-robot control for target tracking with onboard sensing", The International Journal of Robotics Research, 34(13), pp. 1660-1677 (2015).

13. Nagaty, A., Thibault, C., Trentini, M., and Li, H. "Probabilistic cooperative target localization", IEEE Transactions on Automation Science and Engineering, 12(3), pp. 786-794 (2015).

14. Koohifar, F., Guvenc, I., and Sichitiu, M.L., Autonomous Tracking of Intermittent RF Source Using a UAV Swarm, IEEE Access (2018).

15. Shin, H.S., Garcia, A.J., and Alvarez, S. "Informationdriven persistent sensing of a non-cooperative mobile target using UAVs", Journal of Intelligent \& Robotic Systems, 92(3-4), pp. 629-643 (2018).

16. Chakraborty, A., Taylor, C.N., Sharma, R., and Brink, K.M. "Cooperative localization for fixed wing unmanned aerial vehicles", In IEEE/ION Position, Location and Navigation Symposium (PLANS), IEEE, pp. 106-117 (2016). 
17. Bourgault, F., Furukawa, T., and Durrant-Whyte, H.F. "Coordinated decentralized search for a lost target in a Bayesian world", Intelligent Robots and Systems (IROS 2003), Proceedings IEEE/RSJ International Conference on, 1, IEEE (2003).

18. Wang, X., Zhu, H., Zhang, D., Zhou, D., and Wang, $\mathrm{X}$. "Vision-based detection and tracking of a mobile ground target using a fixed-wing UAV", International Journal of Advanced Robotic Systems, 11 p. 156 (2014).

19. Pirshayan, A., Seyedarabi, H., and Haghipour, S. "Target localization using cooperative unmanned aerial vehicles", Advances in Computer Science: An International Journal, 3(4), pp. 68-73 (2014).

20. Ponda, S., Kolacinski, R., and Frazzoli, E., Trajectory Optimization for Target Localization Using Small Unmanned Aerial Vehicles, Diss. Massachusetts Institute of Technology, Department of Aeronautics and Astronautics (2008).

21. York, G. and Pack, D.J. "Ground target detection using cooperative unmanned aerial systems", Journal of Intelligent \& Robotic Systems, 65(1-4) pp. 473-478 (2012).

22. Dogancay, K. "UAV path planning for passive emitter localization", Aerospace and Electronic Systems, IEEE Transactions on, 48(2), pp. 1150-1166 (2012).

23. Esmailifar, S.M. and Saghafi, F. "A guidance based Algorithm for multiple flying vehicle search", 28th International Congress of the Aeronautical Sciences, (Sep. 2012).

24. Esmailifar, S. and Saghafi, F. "Moving target localization by cooperation of multiple flying vehicles", Aerospace and Electronic Systems, IEEE Transactions on, 51(1), pp. 739-746 (2015).

25. Esmailifar, S.M. and Saghafi, F. "Development and stability analysis of a cooperative search algorithm by multiple flying vehicles", Proceedings of the Institution of Mechanical Engineers, Part G: Journal of Aerospace Engineering, 228(7), pp. 1058-1075 (2014).

26. Morris, K.M., Mullins, B.E., Pack, D.J., York, G.W., and Baldwin, R.O. "Impact of limited communications on a cooperative search algorithm for multiple UAVs." Networking, Sensing and Control, 2006, ICNSC'06, Proceedings of the 2006 IEEE International Conference on, IEEE (2006).

27. Pack, D.J. and York, G.W. "Developing a control architecture for multiple unmanned aerial vehicles to search and localize RF time-varying mobile targets: Part I", Robotics and Automation, ICRA Proceedings of the IEEE International Conference on, IEEE (2005).

28. Pack, D. and York, G. "An extended time horizon search technique for cooperative unmanned vehicles to locate mobile RF targets", Conference on Collaborative Technologies and Systems, pp. 333-338 (2005).

29. Pack, D. and York, G. "An extended time horizon search technique for cooperative unmanned vehicles to locate mobile RF targets", Collaborative Technologies and Systems, Proceedings of the International Symposium on, IEEE (2005).

30. Pack, D.J., DeLima, P., Toussaint, G.J., and York, G. "Cooperative control of UAVs for localization of intermittently emitting mobile targets", Systems, Man, and Cybernetics, Part B: Cybernetics, IEEE Transactions on, 39(4), pp. 959-970 (2009).

31. Pack, D., York, G., and Toussaint, G. "Localizing mobile RF targets using multiple unmanned aerial vehicles with heterogeneous sensing capabilities", Networking, Sensing and Control, Proceedings IEEE (2005).

32. Toussaint, G.J., De Lima, P., and Pack, D.J. "Localizing RF targets with cooperative unmanned aerial vehicles", American Control Conference, ACC'07, IEEE (2007).

33. Plett, G., DeLima, P., and Pack, D. "Target localization using multiple UAVs with sensor fusion via sigmapoint Kalman filtering", Proceedings of the AIAA (2007).

34. Hager, C., Zarzhitsky, D., Kwon, H., and Pack, D. "Cooperative target localization using heterogeneous unmanned ground and aerial vehicles", Intelligent Robots and Systems (IROS), IEEE/RSJ International Conference on, IEEE (2010).

35. Nobahari, H. and Pourtakdoust, S.H. "An optimalfuzzy two-phase CLOS guidance law design using ant colony optimization", Aeronautical Journal, 111(1124), pp. 621-636 (2007).

36. Hedrick, J.K. and Girard, A. "Control of nonlinear dynamic systems: Theory and applications", Controllability and observability of Nonlinear Systems (2005).

37. Kalman, R.E. "On the general theory of control systems", In Proceedings First International Conference on Automatic Control, Moscow, USSR (1960).

38. James, M.R. "Controllability and observability of nonlinear systems", Mathematics Department and Systems Research Center, University of Maryland, College Park, MD 20742, USA, October (1986).

39. Jazwinski, A.H., Stochastic Processes and Filtering Theory, Academic Press, New York (1970).

40. Ristic, B., Arulampalam, S., and Gordon, N. "Beyond the Kalman filter", IEEE Aerospace and Electronic Systems Magazine, 19(7), pp. 37-38 (2007).

\section{Appendix}

\section{Observability analysis}

Definition. A nonlinear system is called locally $o b$ servable at a point $\mathbf{x}_{0} \in X$, if there are sufficient numbers of linearly independent vectors in the gradients of Lie derivatives evaluated at $\mathbf{x}_{0}$. It means that $\mathbf{O}\left(\mathbf{x}_{0}, \mathbf{u} *\right)=\partial \mathbf{l}(\mathbf{x}) /\left.\partial \mathbf{x}\right|_{\mathbf{x}_{0}}$ must be full rank, where $\mathbf{I}$ is 
Lie derivative and is defined as follows:

$$
\mathbf{l}\left(\mathbf{x}_{0}, \mathbf{u} *\right) \equiv\left[\begin{array}{c}
L_{f}^{0}\left(h_{1}\right) \\
\ldots \\
L_{f}^{0}\left(h_{p}\right) \\
\ldots \\
L_{f}^{n-1}\left(h_{1}\right) \\
\ldots \\
L_{f}^{n-1}\left(h_{p}\right)
\end{array}\right]
$$

where $L_{f}$ is Lie derivative with respect to $f$ and is defined as follows:

$$
\begin{aligned}
L_{f} h & =\nabla h . f=\frac{\partial h}{\partial x} . f=\sum_{i=1}^{n} \frac{\partial h}{\partial x_{i}} f_{i} \\
& =\left[\frac{\partial h}{\partial x_{1}}, \ldots, \frac{\partial h}{\partial x_{n}}\right]\left[\begin{array}{c}
f_{1} \\
f_{n}
\end{array}\right] .
\end{aligned}
$$

Relative equations of Eq. set (A.3) are utilized for the localization of ground targets by EKF.

$\ddot{x}_{T}-\ddot{x}_{U}=-a_{x, U} \cos \theta_{U} \cos \psi_{U}+a_{z, U}$

$$
\left(\cos \phi_{U} \sin \theta_{U} \cos \psi_{U}-\sin \phi_{U} \sin \psi_{U}\right)
$$

$\ddot{y}_{T}-\ddot{y}_{U}=-a_{x, U} \cos \theta_{U} \sin \psi_{U}+a_{z, U}$

$$
\left(\cos \phi_{U} \sin \theta_{U} \sin \psi_{U}-\sin \phi_{U} \cos \psi_{U}\right)
$$

Moreover, Eq. (A.3) in the state space form is as:

$$
\begin{aligned}
\dot{x}_{1}= & x_{2}, \\
\dot{x}_{2}= & -a_{x, U} \cos \theta_{U} \cos \psi_{U}+a_{z, U} \\
& \left(\cos \phi_{U} \sin \theta_{U} \cos \psi_{U}-\sin \phi_{U} \sin \psi_{U}\right), \\
\dot{x}_{3}= & x_{4}, \\
\dot{x}_{4}= & -a_{x, U} \cos \theta_{U} \sin \psi_{U}+a_{z, U} \\
& \left(\cos \phi_{U} \sin \theta_{U} \sin \psi_{U}-\sin \phi_{U} \cos \psi_{U}\right) .
\end{aligned}
$$

Now, observability of the equations will be investigated. According to Eqs. (29), (30), and (31), the following equations are obtained:

$$
\begin{aligned}
& h_{1}=\varepsilon=\tan ^{-1}\left(\frac{z_{r}}{x_{r}}\right)=\tan ^{-1}\left(\frac{\Delta h}{x_{1}}\right), \\
& h_{2}=\sigma=\tan ^{-1}\left(\frac{y_{r}}{x_{r}}\right)=\tan ^{-1}\left(\frac{x_{3}}{x_{1}}\right) .
\end{aligned}
$$

Moreover, using Eqs. (A.2), (A.5), and (A.6), the following equations are calculated:

$$
\begin{aligned}
L_{f} h_{1} & =x_{2} \frac{\partial \tan ^{-1}\left(\frac{\Delta h}{x_{1}}\right)}{\partial x_{1}}, \\
L_{f} h_{2} & =x_{2} \frac{\partial \tan ^{-1}\left(\frac{x_{3}}{x_{1}}\right)}{\partial x_{1}}+x_{4} \frac{\partial \tan ^{-1}\left(\frac{x_{3}}{x_{1}}\right)}{\partial x_{3}} .
\end{aligned}
$$

Therefore, the observability matrix, $\mathbf{O}$, is calculated as follows:

$$
\begin{gathered}
\mathbf{O}=\left[\begin{array}{llll}
d h_{1} & d h_{2} & d L_{f}\left(h_{1}\right) & d L_{f}\left(h_{2}\right) \\
& \ldots \quad d L_{f}^{3}\left(h_{1}\right) & d L_{f}^{3}\left(h_{2}\right)
\end{array}\right]_{1 \times 8}^{T} .
\end{gathered}
$$

By using the code, provided in Table A.1, the observability matrix is obtained as follows:

$$
\mathbf{O}=\left[\begin{array}{cccc}
m_{1} & 0 & 0 & 0 \\
m_{2} & 0 & m_{3} & 0 \\
m_{4} & m_{5} & 0 & 0 \\
m_{6} & m_{7} & m_{8} & m_{9} \\
m_{10} & m_{11} & 0 & 0 \\
m_{12} & m_{13} & m_{14} & m_{15} \\
m_{16} & m_{17} & 0 & 0 \\
m_{18} & m_{19} & m_{20} & m_{21}
\end{array}\right] .
$$

By defining $f_{1}$ and $f_{2}$ as:

$$
f_{1}=-a_{x, U} \cos \theta_{U} \cos \psi_{U}+a_{z, U}
$$$$
\left(\cos \phi_{U} \sin \theta_{U} \cos \psi_{U}-\sin \phi_{U} \sin \psi_{U}\right),
$$

$$
\begin{aligned}
f_{2}= & -a_{x, U} \cos \theta_{U} \sin \psi_{U}+a_{z, U} \\
& \left(\cos \phi_{U} \sin \theta_{U} \sin \psi_{U}+\sin \phi_{U} \cos \psi_{U}\right) .
\end{aligned}
$$

The elements of the observability matrix are stated as follows:

$$
\begin{aligned}
& m_{1}=-\frac{\Delta h}{\Delta h^{2}+x_{1}^{2}}, \\
& m_{2}=-\frac{x_{3}}{x_{1}^{2}+x_{3}^{2}}, \\
& m_{3}=\frac{x_{1}}{x_{1}^{2}+x_{3}^{2}}, \\
& m_{4}=\frac{2 \Delta h x_{1} x_{2}}{\left(\Delta h^{2}+x_{1}^{2}\right)^{2}}, \\
& m_{5}=-\frac{\Delta h}{\Delta h^{2}+x_{1}^{2}}, \\
& m_{6}=\frac{-x_{4} x_{1}^{2}+2 x_{2} x_{1} x_{3}+x_{4} x_{3}^{2}}{\left(x_{1}^{2}+x_{3}^{2}\right)^{2}},
\end{aligned}
$$


Table A.1. Written code in MATLAB MuPAD to calculate the observability matrix and its rank.

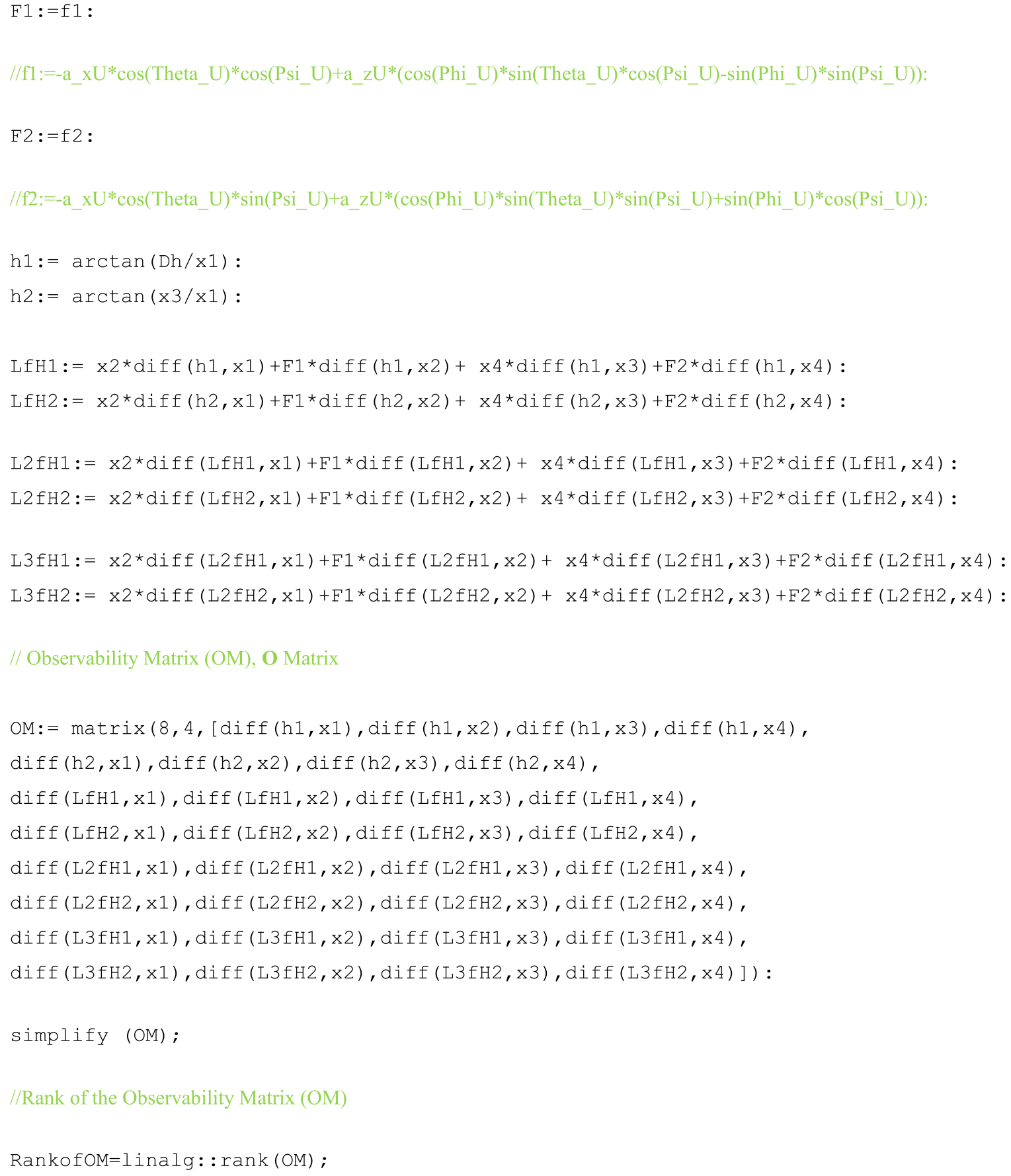

$m_{7}=-\frac{x_{3}}{x_{1}^{2}+x_{3}^{2}}$

$m_{8}=-\frac{x_{2} x_{1}^{2}+2 x_{4} x_{1} x_{3}-x_{2} x_{3}^{2}}{\left(x_{1}^{2}+x_{3}^{2}\right)^{2}}$,

$m_{9}=\frac{x_{1}}{x_{1}^{2}+x_{3}^{2}}$,

$$
m_{10}=\frac{2 \Delta h\left(f_{1} \Delta h^{2} x_{1}+\Delta h^{2} x_{2}^{2}+f_{1} x_{1}^{3}-3 x_{1}^{2} x_{2}^{2}\right)}{\left(\Delta h^{2}+x_{1}^{2}\right)^{3}}
$$




$$
\begin{aligned}
& m_{13}=\frac{2\left(-x_{4} x_{1}^{2}+2 x_{2} x_{1} x_{3}+x_{4} x_{3}^{2}\right)}{\left(x_{1}^{2}+x_{3}^{2}\right)^{2}}, \\
& m_{14}=-\frac{f_{1} x_{1}^{4}-2 x_{1}^{3} x_{2}^{2}+2 f_{2} x_{1}^{3} x_{3}+\ldots}{\left(x_{1}^{2}+x_{3}^{2}\right)^{3}}, \\
& m_{15}=-\frac{2\left(x_{2} x_{1}^{2}+2 x_{4} x_{1} x_{3}-x_{2} x_{3}^{2}\right)}{\left(x_{1}^{2}+x_{3}^{2}\right)^{2}}, \\
& m_{16}= \\
& -\frac{6 \Delta h x_{2}\left(-f_{1} \Delta h^{4}+2 f_{1} \Delta h^{2} x_{1}^{2}+4 \Delta h^{2} x_{1} x_{2}^{2}+\ldots\right)}{\left(\Delta h^{2}+x_{1}^{2}\right)^{4}} \\
& m_{17}=\frac{6 \Delta h\left(f_{1} \Delta h^{2} x_{1}+\Delta h^{2} x_{2}^{2}+f_{1} x_{1}^{3}-3 x_{1}^{2} x_{2}^{2}\right)}{\left(\Delta h^{2}+x_{1}^{2}\right)^{3}} \\
& m_{18}=-\frac{6\left(-f_{2} x_{1}^{5} x_{2}-f_{1} x_{1}^{5} x_{4}+3 x_{1}^{4} x_{2}^{2} x_{4}+\ldots\right)}{\left(x_{1}^{2}+x_{3}^{2}\right)^{4}}, \\
& m_{19}=\frac{3\left(-f_{2} x_{1}^{4}+4 x_{1}^{3} x_{2} x_{4}+2 f_{1} x_{1}^{3} x_{3}+\ldots\right)}{\left(x_{1}^{2}+x_{3}^{2}\right)^{3}}, \\
& m_{20}=\frac{6\left(f_{1} x_{1}^{5} x_{2}-f_{2} x_{1}^{5} x_{4}-x_{1}^{4} x_{2}^{3}+\ldots\right)}{\left(x_{1}^{2}+x_{3}^{2}\right)^{4}}, \\
& m_{21}=-\frac{3\left(f_{1} x_{1}^{4}-2 x_{1}^{3} x_{2}^{2}+2 f_{2} x_{1}^{3} x_{3}+\ldots\right)}{\left(x_{1}^{2}+x_{3}^{2}\right)^{3}} .
\end{aligned}
$$

By using Table A.1, the rank of $\mathbf{O}$ is 4 , if $x_{1}^{2}+\Delta h^{2} \neq 0$ and $x_{1}^{2}+x_{3}^{2} \neq 0$; therefore, $\mathbf{O}$ is full rank and the system is fully observable, provided that $x_{r}^{2}+\Delta h^{2} \neq 0$ and $x_{r}^{2}+y_{r}^{2} \neq 0$. The first condition means that the relative longitudinal position and the relative altitude of the UAV with respect to the target must not be zero, simultaneously. The second condition means that the relative longitudinal and lateral positions of the UAV with respect to the target must not be zero, simultaneously. It should be mentioned that $x_{r}^{2}+\Delta h^{2}=0$ is physically impossible, because the altitude of the UAV differs from that of the target. In addition, $x_{r}^{2}+y_{r}^{2}=0$ is also impossible, because, during the LT mode, the UAV performs a circular motion around the target.

\section{Biographies}

Hadi Nobahari is an Associate Professor at the Department of Aerospace Engineering at Sharif University of Technology. He received his BS, MS, and $\mathrm{PhD}$ in Aerospace Engineering, Flight Dynamic and Control Division, from Sharif University of Technology in 1998, 2000, and 2006, respectively. He is now the Director of Guidance and Control Research Center. His research interests include novel applications of heuristic algorithms in aerospace, intelligent guidance and control systems, and cooperative guidance and navigation systems.

Meysam Effati received his BS degree in Aerospace Engineering from Sharif University of Technology, Tehran, Iran in 2010. In addition, he received his MS degree with honors in Flight Dynamics and Control from Sharif University of Technology in 2014. Now, he is a $\mathrm{PhD}$ applicant. His research interests include robotics, modeling and control of robots, multi-agent coordination, swarm robotics, and system identification.

Mahyar Motie received his BS degree in Aerospace Engineering from Amirkabir University of Technology, Tehran, Iran, and his MS degree in Flight Dynamic and Control from Sharif University of Technology. He has been working as a Research Assistant at Guidance and Control Research Center since 2011. His fields of research include guidance algorithm, cooperation and task allocation between multiple vehicles, real-time implementation, and optimization. 